\title{
Nondeterministic Manifest Contracts
}

\author{
Yuki Nishida \\ Graduate School of Informatics \\ Kyoto University \\ nishida@fos.kuis.kyoto-u.ac.jp
}

\author{
Atsushi Igarashi \\ Graduate School of Informatics \\ Kyoto University \\ igarashi@kuis.kyoto-u.ac.jp
}

\begin{abstract}
We study a manifest contract system-a typed calculus of higherorder contracts where contracts are tightly integrated into a refinement type system-for a functional language with nondeterministic choice. The extension is not trivial, especially in the presence of dependent function types, because a naive extension would lead to inconsistent type equivalence, which makes contract information in refinement types meaningless.

To solve the problem, we propose a new kind of nondeterministic choice called coordinated choice, in which each occurrence of a choice operator is given a name and choices of the same name coordinately take the same branch. We introduce the notion of orthant that helps both intuitive understanding and the development of formal semantics of the new choice.

We formalize a manifest contract system $\lambda^{\mathrm{H} \|^{\Phi}}$ using the coordinated choice and show its basic properties of progress, type preservation, and contract satisfaction, the last of which states correctness of contracts in refinement types.
\end{abstract}

\section{CCS CONCEPTS}

- Theory of computation $\rightarrow$ Operational semantics; Lambda calculus; • Software and its engineering $\rightarrow$ Functional languages; Formal software verification;

\section{ACM Reference Format:}

Yuki Nishida and Atsushi Igarashi. 2018. Nondeterministic Manifest Contracts. In The 20th International Symposium on Principles and Practice of Declarative Programming (PPDP '18), September 3-5, 2018, Frankfurt am Main, Germany. ACM, New York, NY, USA, 13 pages. https://doi.org/10. $1145 / 3236950.3236964$

\section{INTRODUCTION}

\subsection{Nondeterministic programming}

Nondeterminism is a powerful tool for programming. It is especially useful when we know what we are going to implement but not how we do. For example, consider implementing a function which takes a list of natural numbers and returns a pair of natural numbers which are taken from the list and whose sum is a prime number.

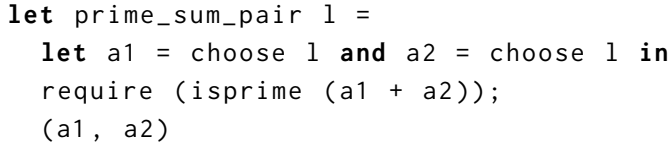

Permission to make digital or hard copies of part or all of this work for personal or classroom use is granted without fee provided that copies are not made or distributed for profit or commercial advantage and that copies bear this notice and the full citation on the first page. Copyrights for third-party components of this work must be honored For all other uses, contact the owner/author(s).

PPDP '18, September 3-5, 2018, Frankfurt am Main, Germany

(c) 2018 Copyright held by the owner/author(s).

ACM ISBN 978-1-4503-6441-6/18/09.

https://doi.org/10.1145/3236950.3236964
Here, choose returns an element of a given list nondeterministically, require $M$ aborts an execution unless the condition $M$ holds, and isprime is a Boolean function that returns whether its argument is prime or not. So, the defined function nondeterministically returns a pair of the expected property. A remarkable point is that we are almost free from considering how to choose proper elements from the given list and just write down the specifications into the code.

A well-known approach to bringing nondeterminism to a functional language is the introduction of a choice operator, which we write $(M \| N)$ in this paper. It takes two operands $M, N$ and returns one of them nondeterministically. Using the choice, choose function we have seen in the example above can be defined as follows.

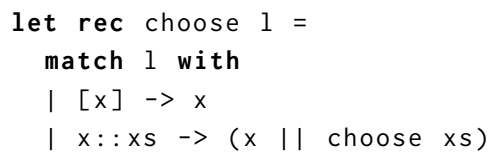

To deal with the case where choose is called with an empty list, one may want to insert require (not (isempty 1 )) before match.

\subsection{Software contracts and manifest contract systems}

In this paper, we study software contracts, advocated by Meyer [Meyer 1988] with his famous methodology "design by contract", in a nondeterministic programming language. In particular, we focus on manifest contracts [Greenberg et al. 2010] as a vehicle of study.

Manifest contract systems [Belo et al. 2011; Flanagan 2006; Greenberg 2015; Greenberg et al. 2010; Gronski et al. 2006; Knowles and Flanagan 2010; Sekiyama and Igarashi 2017; Sekiyama et al. 2017, 2015; Wadler and Findler 2009], which originally arise as a formal basis of hybrid type checking [Flanagan 2006; Knowles and Flanagan 2010], are typed functional calculi of software contracts, where contracts are tightly integrated into their type systems. A contract appears in a refinement type of the form $\{x: \tau \mid M\}$; it represents the subset of the underlying type $\tau$ where the values in the subset satisfy the predicate $M$, which can be an arbitrary Boolean expression. Using refinement types, we can express specifications as types: For example, the expected behavior of prime_sum_pair can be expressed by type int list $\rightarrow\{x$ :int $\times$ int $\mid$ isprime (fst $x+$ snd $x)\}$.

In addition to refinement types, manifest contract systems usually provide dependent function types to express the relationship between an input and output of a function. A dependent function takes the form $(x: \sigma) \rightarrow \tau$ and represents a function that takes an argument of type $\sigma$ and returns a value of type $\tau$; the return type $\tau$ actually depends on the actual argument represented by $x$. For example, a function of type $(x:$ int $) \rightarrow\{z$ :int $\mid x=z\}$ has to return an integer which is equal to the argument $x$. So, it represents the type of the identity function on integers. 
In a manifest contract calculus, contracts are dynamically checked ${ }^{1}$ by explicit casts, that is, type conversion of the form $(M: \sigma \triangleright \tau)$. We call $M$ the subject, $\sigma$ the source type, and $\tau$ the target type of the cast. A cast checks whether $M$, whose static type is $\sigma$, can have type $\tau$ and, if the check fails, it signals an uncatchable exception called blame, which stands for contract violation. Although manifest contract systems are not strong enough to guarantee the absence of contract violation statically, they do guarantee that the result of a successful execution of a program satisfies the refinement predicates in the program's type. This property follows from type preservation and a property called value inversion [Sekiyama et al. 2015]-if a value $V$ has a refinement type $\{x: \tau \mid M\}$, the expression obtained by substituting $V$ for $x$ in $M$ is always evaluated to true.

We believe that it is meaningful to introduce manifest contracts into a nondeterministic language. Let us revisit the example of prime_sum_pair. By using casts, prime_sum_pair could be written as follows:

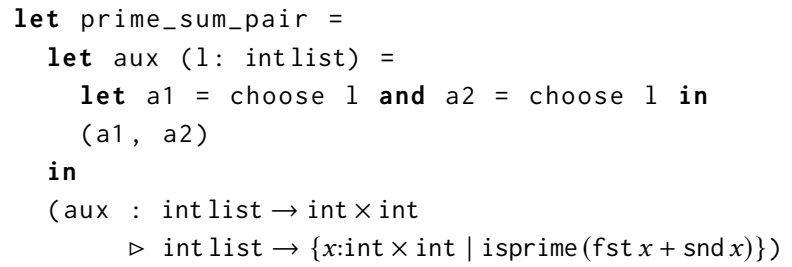

The type of prime_sum_pair, which is the target type of the cast on aux, is as expected.

Note that the cast on aux is a higher-order cast, in which the source and target types are function types. The semantics of such higher-order casts is similar to higher-order contract checking by Findler and Felleisen [2002] (although there are subtle differencessee [Greenberg et al. 2010]). Roughly speaking, checking is delayed until aux is applied to an argument and, when an argument is given, the argument is checked by a cast from the domain type int list of the target type (of the higher-order cast) to int list, that of the source type, and then passed to aux; finally, the value returned from aux is checked by a cast from the codomain type int $x$ int of the source type to $\{x$ :int $\times$ int $\mid$ isprime $($ fst $x+$ snd $x)\}$, that of the target type. The cast on the argument is trivial and the cast on the return value amounts to checking the returned integer pair $x$ satisfies isprime (fst $x+\operatorname{snd} x$ ), which is, in fact, what require does in the first version.

An interesting (and perhaps controversial) point in the design space is that whether we should allow nondeterminism in predicates in refinement types. We would like to allow it so that any library function, which may be nondeterministic, can be used to describe predicates.

\subsection{Our work}

This paper develops a formal calculus $\lambda^{\mathrm{H} \|^{\Phi}}$ of manifest contracts with a nondeterministic choice operator. We follow the subsumptionfree approach [Belo et al. 2011] to manifest contracts, in which all type conversions are made explicit with casts, in order to simplify the calculus and metatheory. Among variants of the semantics for choice [Søndergaard and Sestoft 1992], we will investigate

${ }^{1}$ As we have mentioned, manifest contracts emerged from the context of hybrid type checking, which integrates static and dynamic contract verification [Flanagan 2006]. the so-called singular semantics, ${ }^{2}$ which corresponds to call-time choice [Hennessy and Ashcroft 1977], because we think it is easy to understand. This semantics also poses interesting technical challenges, as we will discuss shortly. The goal of the present paper is to prove basic meta-theoretic properties of progress, type preservation (subject reduction) [Wright and Felleisen 1994], and contract satisfaction, which is a generalization of value inversion and means that, if $M$ is given type $\{x: \tau \mid N\}$, then the result of any successful execution of $M$ satisfies predicate $N$ nondeterministically.

As far as a simple program like prime_sum_pair is concerned, nondeterministic choice seems to integrate smoothly into a manifest contract system. In fact, it would integrate smoothly, if it were not for dependent function types.

However, the combination of dependent function types and nondeterministic choice-in particular, call-time choice-poses a few technical challenges. Before discussing them, let us recall the typing rule for function applications, which is standard in a language with dependent function types:

$$
\frac{\Gamma \vdash M:\left(x: \tau_{1}\right) \rightarrow \tau_{2} \quad \Gamma \vdash N: \tau_{1}}{\Gamma \vdash M N: \tau_{2}[x:=N]}
$$

The first challenge is how to define type equivalence, which is used to show type preservation. Dependent type systems have a type equivalence relation $\equiv$ and a typing rule that allows the type of a term to be converted to an equivalent one, so that the two types $\tau_{2}[x:=N]$ and $\tau_{2}\left[x:=N^{\prime}\right]$ where $N \longrightarrow N^{\prime}$ are related and thereby a reduction step $V N \longrightarrow V N^{\prime}$, where $V$ is a value of type $\left(x: \tau_{1}\right) \rightarrow \tau_{2}$, preserves the type. In (subsumption-free) manifest contract calculi, their type equivalence relations are derived from term equivalence, which includes the reduction relation. However, standard reduction rules for choice

$$
(M \| N) \longrightarrow M \quad(M \| N) \longrightarrow N
$$

would make type equivalence inconsistent because term equivalence closed under these rules would relate any two expressions $M$ and $N$ through $M \longleftarrow(M \| N) \longrightarrow N$. Such inconsistency would break value inversion because any refinement type $\{x: \tau \mid M\}$ can be converted to $\{x: \tau \mid$ false $\}$.

Another challenge is how to settle down interaction between the singular semantics and substitution $\tau_{2}[x:=N]$ that appears in the typing rule for applications above. It duplicates possibly nondeterministic computation $N$. However, such duplication is contradictory to the expectation in the singular semantics that $x$ has a single value. One solution to the problem would be to restrict predicates of refinement types to pure ones, that is, predicates has no nondeterminism. Although the restriction seems reasonable and could be easily accomplished [Unno et al. 2018], we avoid such a restriction to give programmers full expressiveness in writing software contracts.

To address the two challenges above, we give a new kind of choice, called coordinated choice. For the former, the choice does not discard an alternative, but reduction retains the set of all possible executions as in Kutzner and Schmidt-Schauß [1998]. For the

\footnotetext{
${ }^{2}$ Roughly speaking, in the singular semantics, every occurrence of a variable of the same name in the same scope yields the same value, although the value itself may be chosen nondeterministically. For example, the value of $(\lambda x \cdot x+x)(1 \| 2)$ is either 2 or 4 .
} 
latter, different occurrences of choices can share nondeterministic decisions via names; thus the semantics stays singular even though syntactic duplication of a choice takes place.

Our contributions are summarized as follows:

- we propose a new kind of nondeterministic choice, called coordinated choice;

- we formalize an operational semantics of coordinated choice;

- we formalize $\lambda^{\mathrm{H} \|^{\Phi}}$, a nondeterministic manifest contract system by using coordinated choice; and

- we state progress, type preservation, and contract satisfaction and sketch their proofs.

We omit detailed proofs. Interested readers are referred to a full version at http://www.fos.kuis.kyoto-u.ac.jp/ igarashi/papers/pdf/ nmc-full.pdf.

Outline of the rest of the paper. Firstly, in Section 2, we introduce coordinated choice. Then, in Section 3, we integrate the new choice into a manifest contract system. In Section 4, we discuss the basic properties and sketch their proofs. We discuss related work in Section 5 and conclude in Section 6.

\section{COORDINATED CHOICE}

As we have discussed in Section 1, the combination of dependent function types and nondeterministic choice, in particular, naive call-time choice, poses two challenges on manifest contracts. In this section, we informally describe our solution.

The first problem of inconsistency of type equivalence is avoided by not using the naive rules to choose one branch and, instead, introducing rules that distribute an evaluation context over choices [Kutzner and Schmidt-Schauß 1998], such as

$$
V\left(M_{1} \| M_{2}\right) \longrightarrow\left(V M_{1} \| V M_{2}\right)
$$

(where $V$ stands for a value). For example, $(\lambda x . x)(1 \| 2)$ reduces to $(1 \| 2)$ via $((\lambda x . x) 1 \|(\lambda x . x) 2)$. Similarly, $(1 \| 2)+(3 \| 4)$ reduces to $((4|| 5) \|(5 \| 6))$. So, our reduction relation expresses all possible nondeterministic executions at once.

The second problem of the interaction between the singular semantics and substitution is addressed by coordination of choices. Actually, our choice is given a name $\Phi$ and written $\left(M \|{ }^{\Phi} N\right)$; the name can be shared by another choice. The choices of the same name "synchronize," that is, globally make the same decision: For example, expression $\left(1 \|^{\text {foo }} 2\right)+\left(3 \|^{\text {bar }} 4\right)$ (here foo and bar are names) nondeterministically evaluates to 4,5 , or 6 , whereas $\left(1 \|^{\text {foo }} 2\right)+\left(3 \|^{\text {foo }} 4\right)$ cannot evaluate to 5 because if 1 is chosen by the first choice, 3 must be chosen by the other.

To accomplish the coordination, we will introduce rules to discard impossible results. For example, $\left(1 \|^{\text {foo }} 2\right)+\left(3 \|^{\text {foo }} 4\right)$ reduces to $\left(\left(4 \|^{\text {foo }} 5\right) \|^{\text {foo }}\left(5 \|^{\text {foo }} 6\right)\right)$ (via distribution of evaluation contexts), but the two occurrences of 5 are bogus. Actually, we can see that they are bogus only from the syntactic structure of an expression: a subexpression is bogus if it is reached by choosing different sides at choices of the same name. In the example above, the first occurrence of 5 is bogus because it is reached by first taking the left side of $\|^{\text {foo }}$ and then the right of $\|^{\text {foo }}$ Although the general rule to discard one branch of a choice is not allowed, our reduction relation does allow a bogus expression to be discarded; so, $\left(\left(4 \|^{\text {foo }} 5\right) \|^{\text {foo }}\left(5 \|^{\text {foo }}\right.\right.$ 6)) reduces to $\left(4 \|^{\text {foo }} 6\right)$ in two steps, whereas $\left(1 \|^{\text {foo }} 2\right)+\left(3 \|^{\text {bar }} 4\right)$ reduces to $\left(\left(4 \|^{\text {foo }} 5\right) \|^{\text {bar }}\left(5 \|^{\text {foo }} 6\right)\right)$, which is normal.

Somewhat surprisingly, these two ideas are sufficient for basic correctness properties to hold of a manifest contract system with nondeterministic choice.

To formalize the reduction relation as described above, we introduce the notion of orthant, inspired by geometry.

\subsection{Orthant}

As we have described, an expression evaluates to a set of values (combined by $\|^{\Phi}$ ). We view each element in the set inhabit an "orthant," generated by splitting the whole space by axes corresponding to names on choices.

Figure 1 shows an informal interpretation of expressions. The first example shows a basic interpretation: the expression $\left(1 \|^{\Phi}\right.$ $2)+\left(3 \|^{\Psi} 4\right)$ (where $\left.\Phi \neq \Psi\right)$ represents four expressions since the names of the choices are different. Furthermore, each expression inhabits a different orthant, which is denoted by a set of signed names $\left(\Phi_{+}\right.$, meaning the left side of $\|^{\Phi}$, or $\Phi_{-}$, meaning the right): For instance, the expression $1+3$ inhabits the orthant $\left\{\Phi_{+}, \Psi_{+}\right\}$ since 1 is on the left of the choice named $\Phi$ and 3 is on the left of the choice named $\Psi$.

In contrast, the second example shows a case in which choices have the same name. It shows that the expression $\left(1 \|^{\Phi} 2\right)+\left(3 \|^{\Phi} 4\right)$ represents only two expressions since the name is shared.

The third example shows another interpretation-which considers only axis $\Phi$-of the expression in the first example. We do not have to decompose all the choices that occur in an expression, leaving some choices. We can even choose a name that does not occur in an expression. For example, 3, which a simple expression without coordinated choice, splits into 3 that inhabits $\left\{\Phi_{+}\right\}$and 3 that inhabits $\left\{\Phi_{-}\right\}$. Such freedom in the choice of axes is useful to give an interpretation to the fourth example, where a choice is nested only in one branch of a choice.

These interpretations based on orthant-local viewpoints play an important role when we consider formal semantics.

Remark 2.1. Some orthants such as $\left\{\Phi_{+}, \Phi_{-}\right\}$do not really make sense from a geometric point of view. Nevertheless, we allow such orthants for technical reasons (Lemma 4.2). For example, 2 in $\left(\left(1 \|^{\Phi}\right.\right.$ 2) $\left.\|^{\Phi} 3\right)$ is considered to inhabit the nonsensical orthant $\left\{\Phi_{+}, \Phi_{-}\right\}$, which indicates 2 is bogus.

\subsection{Orthant-local reduction}

More formally, we augment the reduction relation with an orthant $\Delta$ and write $M \stackrel{\Delta}{\longrightarrow} N$, which intuitively means " $M$ reduces to $N$ in orthant $\Delta$. . The crux here is that bogus expressions in orthant $\Delta$ can be discarded because it cannot be seen from the $\Delta$-local viewpoint: For example, $\left(1 \|^{\text {foo }} 2\right) \stackrel{\left\{\mathrm{foo}_{+}\right\}}{\longrightarrow} 1$ holds since 2 inhabits $\left\{\mathrm{foo}_{-}\right\}$and is considered bogus in this orthant. Similarly, $\left(1 \|^{\text {foo }}\right.$ 2) $\stackrel{\{\text { foo- }\}}{\longrightarrow} 2$ holds. Thanks to orthant, we can avoid inconsistency of equivalence discussed at the beginning of this section by making 


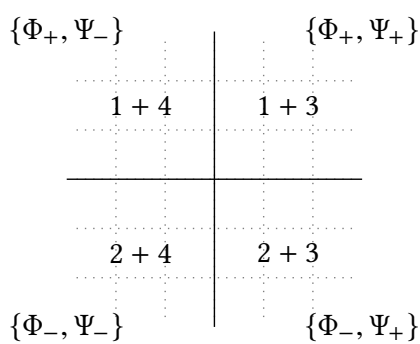

$\left(1 \|^{\Phi} 2\right)+\left(3 \|^{\Psi} 4\right)$

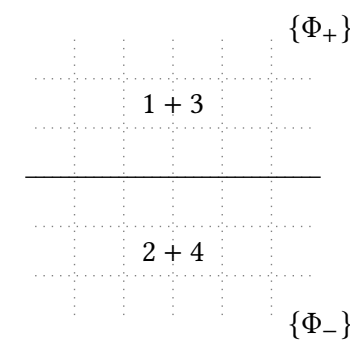

$\left(1 \|^{\Phi} 2\right)+\left(3 \|^{\Phi} 4\right)$

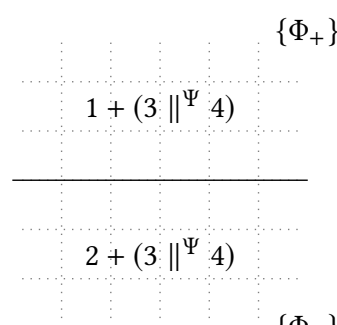

$\left\{\Phi_{-}\right\}$

$\left(1 \|^{\Phi} 2\right)+\left(3 \|^{\Psi} 4\right)$

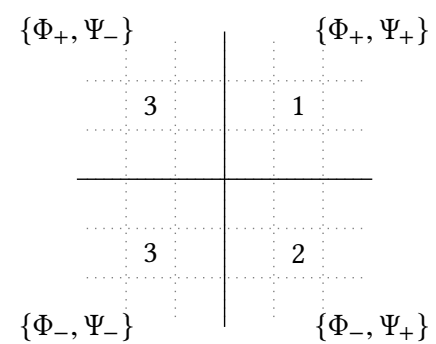

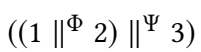

Figure 1: Examples of an informal interpretation of expressions.

the equivalence a family of equivalences indexed by an orthant. ${ }^{3}$ In general, reduction rules that allow discarding bogus expressions can be given as

$$
\left(M \|^{\Phi} N\right) \stackrel{\Delta \cup\left\{\Phi_{+}\right\}}{\longrightarrow} M \quad \text { and } \quad\left(M \|^{\Phi} N\right) \stackrel{\Delta \cup\left\{\Phi_{-}\right\}}{\longrightarrow} N .
$$

Along the same lines, when a subexpression under choice is reduced, the subexpression is reduced in the corresponding orthant. For example, $\left(\left(1 \|^{\text {foo }} 2\right) \|^{\text {foo }} 3\right) \stackrel{\{\}}{\longrightarrow}\left(1 \|^{\text {foo }} 3\right)$ since $\left(1 \|^{\text {foo }} 2\right)$ inhabits the orthant $\left\{\mathrm{foo}_{+}\right\}$and $\left(1 \|^{\mathrm{foo}} 2\right) \stackrel{\left\{\mathrm{foo}_{+}\right\}}{\longrightarrow} 1$. We formalize such reduction by the following two rules:

$$
\frac{M \stackrel{\Delta \cup\left\{\Phi_{+}\right\}}{\longrightarrow} M^{\prime}}{\left(M \|^{\Phi} N\right) \stackrel{\Delta}{\longrightarrow}\left(M^{\prime} \|^{\Phi} N\right)} \frac{N \stackrel{\Delta \cup\left\{\Phi_{-}\right\}}{\longrightarrow} N^{\prime}}{\left(M \|^{\Phi} N\right) \stackrel{\Delta}{\longrightarrow}\left(M \|^{\Phi} N^{\prime}\right)}
$$

Multi-step reduction is written $M \stackrel{\Delta}{\longrightarrow} * N$. Note that the orthant in each reduction step is fixed to $\Delta$. So, $M \stackrel{\Delta}{\longrightarrow} * N$ means $M \stackrel{\Delta}{\longrightarrow}$ $\stackrel{\Delta}{\longrightarrow} N$.

Example 2.2. Now we can see how $\left(1 \|^{\Phi} 2\right)+\left(3 \|^{\Phi} 4\right)$ is evaluated into $\left(4 \|^{\Phi} 6\right)$ in the empty orthant \{\} .

$$
\begin{aligned}
\left(1 \|^{\Phi} 2\right)+\left(3 \|^{\Phi} 4\right) & \stackrel{\{\}}{\longrightarrow} *\left(1+\left(3 \|^{\Phi} 4\right) \|^{\Phi} 2+\left(3 \|^{\Phi} 4\right)\right) \\
& \stackrel{\{\}}{\longrightarrow} *\left(1+3 \|^{\Phi} 2+4\right) \\
& \stackrel{\{\}}{\longrightarrow} *\left(4 \|^{\Phi} 6\right)
\end{aligned}
$$

\subsection{Programming with names}

It is cumbersome to assign appropriate names to choices in a program. Although we believe that in many cases it suffices to assign a fresh name to each choice, choices still coordinate if they are derived from the same program location. To avoid unintentional sharing, we introduce some mechanisms for programming with names below. We hope that name programming discussed below can be automated but leave detailed investigation for future work.

First, we consider the following program.

$$
\begin{aligned}
& 1 \text { let choose } x y=\left(x \|^{\text {foo }} y\right) \text { in } \\
& 2 \quad \text { choose } 12+\text { choose } 34
\end{aligned}
$$

\footnotetext{
${ }^{3}$ Actually, 1 is considered equivalent to 2 (through $\left(1 \|^{\text {foo }} 2\right)$ ) in orthant $\left\{\right.$ foo $_{+}$, foo $\}$ but this orthant is nonsensical-see Remark 2.1 above-meaning both branches are actually bogus.
}

The intention of the function choose is that it returns one of the given arguments nondeterministically. Thus we expect that the program results in three values: 4,5 , and 6 . However, this program does not work as we expect. The result of the program is actually (4 $\|^{\text {foo }}$ 6) because the two calls of choose will result in two choices of the same name and it is not possible for choose to return 2 and 3.

We introduce name abstraction and name application to solve the problem:

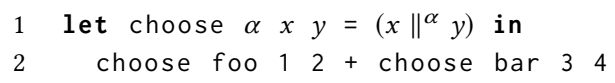

Here, choose takes an additional name parameter $\alpha$, which is used at the choice, and choose is called twice with different names foo and bar. As a result, each call independently returns one of the given arguments, and we can get the expected result.

Unfortunately, when a function is recursive, simple name abstraction and application do not suffice. Consider the following program:

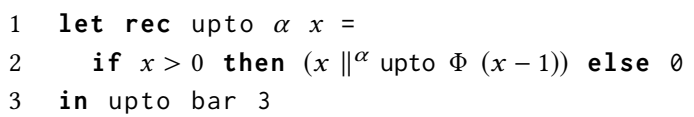

The intention of the function upto is that it returns a random number between 0 and the given argument. Similarly to function choose above, the name of the choice is abstracted as a parameter $\alpha$, which is used in the coordinated choice. Now, the question is what $\Phi$, the name argument to the recursive call to upto, should be. Neither foo nor $\alpha$ is satisfactory because in either case choices of the same name will emerge after a few recursive calls. What we would like here is an ability to create a name that is different from foo and $\alpha$.

We introduce name concatenation $\Phi \Psi$, which yields a name different from $\Phi$ and $\Psi$. Here is the final version of upto:

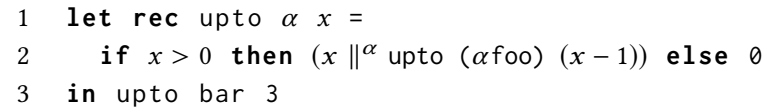

Expanding upto bar 3 will result in $\left(3 \|^{\text {foo }}\left(2 \|^{\text {barfoo }}\left(1 \|^{\text {barfoofoo }}\right.\right.\right.$ $0)$ )), in which the choices do not share their names.

One may wonder why we do not adopt a construct for name generation, found in process calculi such as the $\pi$-calculus [Milner 1999]. Simply put, the main reason is that name generation is another effectful construct and it is not clear how to integrate it into a manifest contract calculus. 


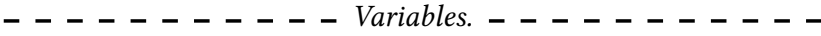

$$
\begin{aligned}
& x, y, z::=\text { term variables } \quad \alpha, \beta::=\text { string variables }
\end{aligned}
$$

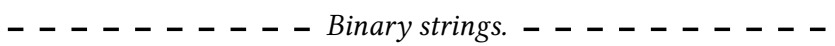

$$
\begin{aligned}
& \Phi, \Psi::=\bullet|\circ| \alpha \mid \Phi \Psi \\
& \text { - - - - - - - - - - Types. - - - - - - - - - - } \\
& \sigma, \tau::=\text { bool } \mid \text { nat }|(x: \sigma) \rightarrow \tau|\{x: \tau \mid M\} \mid \forall \alpha . \tau \\
& \text { - - - - - - - - - Expressions. - - - - - - - - } \\
& L, M, N::=\text { true } \mid \text { false }|0| x|M N| M \Phi \mid \text { fix } f(x: \sigma): \tau . M \\
& |\operatorname{succ}(M)| \operatorname{pred}(M)|\operatorname{iszero}(M)| \text { if } L \text { then } M \text { else } N \\
& |\lambda \alpha \cdot M|\left(M \|^{\Phi} N\right) \mid(M: \sigma \triangleright \tau) \\
& |\langle\langle M ?\{x: \tau \mid N\}\rangle\rangle|\langle\langle M \Rightarrow V:\{x: \tau \mid N\}\rangle\rangle \mid \text { blame } \\
& \text { - - - - - - - - - Values. - - - - - - - - - } \\
& U, V::=\text { true } \mid \text { false }|\bar{n}| \text { fix } f(x: \sigma): \tau . M \mid \lambda \alpha . M \\
& \text { _ _ _ _ _ _ _ Raw results. _ _ _ _ _ _ _ } \\
& R::=V \mid \text { blame } \mid\left(R_{1} \|^{\Phi} R_{2}\right)
\end{aligned}
$$

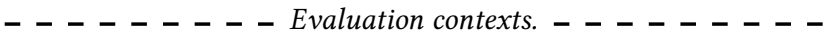

$$
\begin{aligned}
& \mathcal{E}::=\operatorname{succ}([])|\operatorname{pred}([])| \text { iszero([]) | [] } N|V[]|[] \Phi \\
& \text { | if [] then } M \text { else } N \mid([]: \sigma \triangleright \tau) \\
& |\langle\langle[] ?\{x: \tau \mid M\}\rangle\rangle|\langle[[] \Rightarrow V:\{x: \tau \mid M\}\rangle
\end{aligned}
$$

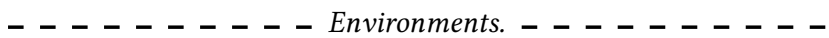

$$
\begin{aligned}
& \Gamma::=\emptyset|\Gamma, \alpha| \Gamma, x: \tau
\end{aligned}
$$

Figure 2: Syntax of $\lambda^{\mathrm{H} \|^{\Phi}}$.

\section{NONDETERMINISTIC MANIFEST CALCULUS $\lambda^{\mathrm{H} \|^{\Phi}}$}

We demonstrate that the coordinated choice actually solves all problems we introduced by first formalizing a manifest contract calculus $\lambda^{\mathrm{H}} \|^{\Phi}$ with coordinated choice in this section and show its soundness properties, including progress, type preservation, and contract satisfaction in the next section. Our calculus $\lambda^{\mathrm{H}} \|^{\Phi}$ is largely based on Sekiyama et al. [2015]; we have removed datatypes and add coordinated choice.

\subsection{Syntax}

The syntax of $\lambda^{\mathrm{H} \|^{\Phi}}$ is shown in Figure 2. We use various metavariables: $x, y, z$ ranging over term variables; $\alpha, \beta$ ranging over string variables; $\Phi, \Psi$ ranging over strings; $\sigma, \tau$ ranging over types, $L, M, N$ ranging over expressions; $U, V$ ranging over values; $R$ ranging over results; $\mathcal{E}$ ranging over (single-frame) evaluation contexts; and $\Gamma$ ranging over type environments. We also use meta-variables primed and/or indexed by number, such as: $x^{\prime}, y^{\prime \prime}, M_{1}$.

Formally (and unlike examples in the last section), names for the choice are sequences of distinguished constants $\bullet$ and $\circ$ and string variables. $\Phi \Psi$ is concatenation of two strings.

The types consist of ground types bool and nat, types for Boolean values and natural numbers, respectively; dependent function types $(x: \sigma) \rightarrow \tau$, in which the term variable $x$ is bound in $\tau$; refinement types $\{x: \tau \mid M\}$, in which the term variable $x$ is bound in predicate
$M$, which is a Boolean expression; and forall types $\forall \alpha . \tau$ for name abstraction, in which the string variable $\alpha$ is bound in $\tau$.

Unlike some manifest contract calculi [Flanagan 2006; Greenberg et al. 2010; Wadler and Findler 2009] that allows only refinement types for ground types, any type, including refinement types, can be refined in $\lambda^{\mathrm{H} \|^{\Phi}}$. For example, $\{x:\{x:$ nat $\mid x \bmod 2=0\} \mid x \bmod$ $2=1\}$, which refines even numbers by the predicate odd numbers satisfy, represents an empty type.

Expressions are usual PCF terms (where abstraction and recursion are integrated into the form $\operatorname{fix} f(x: \sigma): \tau . M)$ extended with string abstraction $\lambda \alpha . M$, in which $\alpha$ is bound in $M$; string application $M \Phi$; coordinated choice $\left(M \|^{\Phi} N\right)$; and casts $(M: \sigma \triangleright \tau)$, which checks dynamically whether the subject $M$ of the source type $\sigma$ can have the target type $\tau$.

There also are run-time expressions, which only occur during reduction and not source programs: waiting check $\langle\langle M ?\{x: \tau \mid$ $N\}\rangle\rangle$, which will check if the subject $M$ satisfies the predicate $N$; active check $\langle\langle M \Rightarrow V:\{x: \tau \mid N\}\rangle\rangle$, which holds an intermediate state $M$ of checking if $V$ satisfies predicate $N$; and blame denoting a cast failure. Leaving blame assignment [Wadler and Findler 2009] for future work, we omit labels to indicate which cast has failed.

The definition of values is standard. Here, $\bar{n}$ is defined as follows.

$$
\bar{n}=\underbrace{\operatorname{succ}(\ldots \operatorname{succ}(0) \ldots)}_{n}
$$

A raw result represents the result of the evaluation of an expression: value $V$; blame; or choice of raw results $\left(R_{1} \|^{\Phi} R_{2}\right)$.

An evaluation context is an expression with a single hole and indicates which subexpression is to be reduced next. We write $\mathcal{E}[M]$ for an expression obtained by replacing the hole in $\mathcal{E}$ with $M$. Note that, unlike standard formulations, the definition is not recursive; a list of our evaluation contexts (which are also called evaluation frames in the literature [Pitts 2000]) corresponds to a standard evaluation context. This definition makes distribution of evaluation contexts over choice deterministic.

A type environment is a sequence of declarations of either a string variable $\alpha$ or a typed variable $x: \tau$. $\emptyset$ denotes the empty type environment. We also use a comma to concatenate environments like $\Gamma_{1}, \Gamma_{2}$. low.

We give a few more definitions and notational conventions be-

Definition 3.1 (Orthant). An orthant, denoted by $\Delta$, is a set of pairs of a string and a symbol + or -, written $\Phi_{+}$or $\Phi_{-}$.

Convention 3.2. We just omit the empty orthant and empty environment if there is no ambiguity.

Convention 3.3. We identify $\alpha$-equivalent expressions, which only differ in their bound variables.

We introduce string substitution $[\alpha:=\Phi]$ of a string for a string variable and substitution $[M:=x]$ of an expression for a variable.

Definition 3.4 (String substitution). String substitution $\Phi[\alpha:=\Psi]$ of $\Psi$ for $\alpha$ in $\Phi$ is defined by:

$$
\begin{array}{ll}
\bullet[\alpha:=\Psi]=\bullet & \circ[\alpha:=\Psi]=\circ \\
\alpha[\alpha:=\Psi]=\Psi & \beta[\alpha:=\Psi]=\beta(\text { if } \alpha \neq \beta) \\
\left(\Phi_{1} \Phi_{2}\right)[\alpha:=\Psi]=\Phi_{1}[\alpha:=\Psi] \Phi_{2}[\alpha:=\Psi]
\end{array}
$$


We extend string substitution to one for orthants by:

$$
\Delta[\alpha:=\Phi]=\left\{\Psi[\alpha:=\Phi]_{+} \mid \Psi_{+} \in \Delta\right\} \cup\left\{\Psi[\alpha:=\Phi]_{-} \mid \Psi_{-} \in \Delta\right\}
$$

and for types (written $\tau[\alpha:=\Phi]$ ) and expressions (written $M[\alpha:=\Phi]$ ) in a straightforward capture-avoiding manner. We extend to type environments $\Gamma[\alpha:=\Phi]$ as follows:

$$
\begin{aligned}
\emptyset[\alpha:=\Phi] & =\emptyset \\
(\Gamma, y: \tau)[\alpha:=\Phi] & =\Gamma[\alpha:=\Phi], y: \tau[\alpha:=\Phi] \\
(\Gamma, \beta)[\alpha:=\Phi] & =\Gamma[\alpha:=\Phi], \beta
\end{aligned}
$$

Definition 3.5 (Substitutions). We define capture-avoiding substitution, written $M[x:=N]$ and $\tau[x:=N]$, of an expression $N$ for a variable $x$ in an expression $M$ and a type $\tau$, respectively, in a usual manner. We extend to type environments $\Gamma[x:=N]$ as follows:

$$
\begin{aligned}
\emptyset[x:=N] & =\emptyset \\
(\Gamma, y: \tau)[x:=N] & =\Gamma[x:=N], y: \tau[x:=N] \\
(\Gamma, \alpha)[x:=N] & =\Gamma[x:=N], \alpha
\end{aligned}
$$

Definition 3.6 (Environment domain and string domain). The domain and string domain of a type environment, written dom $(\Gamma)$ and sdom $(\Gamma)$, respectively, are defined as follows.

$$
\begin{aligned}
\operatorname{dom}(\emptyset) & =\emptyset & \operatorname{sdom}(\emptyset) & =\emptyset \\
\operatorname{dom}(\Gamma, x: \tau) & =\operatorname{dom}(\Gamma) \cup\{x\} & \operatorname{sdom}(\Gamma, x: \tau) & =\operatorname{sdom}(\Gamma) \\
\operatorname{dom}(\Gamma, \alpha) & =\operatorname{dom}(\Gamma) & \operatorname{sdom}(\Gamma, \alpha) & =\operatorname{sdom}(\Gamma) \cup\{\alpha\}
\end{aligned}
$$

Definition 3.7 (Free variables and free string variables). We denote the sets of free variables and free string variables in type $\tau$ by $f \vee(\tau)$ and $f s v(\tau)$, respectively; and denote those in expression $M$ by $f v(M)$ and $f s v(M)$, respectively.

\subsection{Operational semantics}

The reduction relation $M \stackrel{\Delta}{\longrightarrow} N$ is defined between closed expressions $M$ and $N$ because we only consider execution of a program, which is a closed expression. The defining rules are shown in Figure 3. As we discussed in the last section, it defines how an expression is reduced in a specific orthant. The rules are grouped into three: one for PCF expressions; one for casts and dynamic checks; and one for coordinated choice.

Firstly, the rules for PCF expressions are mostly standard. The only exception is pred $(M)$, for which there is no rule to reduce pred (0), so pred (0) is a stuck term. $\lambda^{\mathrm{H} \|^{\Phi}}$ can deal with such a partial function by refinement types, ensuring a well typed program does not reach such a term. (As we can see from the type system, applying pred to a natural number requires a nonzero check in terms of a cast, which may result in blame.)

Secondly, the rules for casts and dynamic checks are adapted from Sekiyama et al. [2015]. (R-CForALL) is new but straightforward: the common foralls are pulled out as string abstraction. Note that the forall-types of $\lambda^{\mathrm{H}} \|^{\Phi}$ are not ones for parametric polymorphism, and thus, this simple reduction rule does not make a problem discussed in Sekiyama et al. [2017]. The side condition $x \neq y$ of (R-CARrow) prevents the inner abstraction (by $y$ ) on the right hand side from capturing the free variable $x$, which must be bound by the outer abstraction, in $\tau_{2}$. Note that there are no free variables other than $x$ and $y$ in $\tau_{2}$ and $\sigma_{2}$ since the reduction relation is defined for closed expressions. The side condition of (R-CPRECHECK) makes cast reduction deterministic-without the condition, it would be possible that both (R-CFORGET) and (R-CPRECHECK) are applied to one expression.

We show a few examples of how a cast is evaluated below.

Example 3.8. The following reduction sequence shows how a simple cast between ground types is executed.

$$
\begin{aligned}
& (1:\{x: \text { nat } \mid x=1\} \triangleright\{x \text { :nat } \mid x>0\}) \\
& \longrightarrow(1: \text { nat } \triangleright\{x \text { :nat } \mid x>0\}) \\
& \longrightarrow\langle\langle(1: \text { nat } \triangleright \text { nat }) ?\{x \text { :nat } \mid x>0\}\rangle\rangle \\
& \longrightarrow\langle\langle 1 ?\{x: \text { nat } \mid x>0\}\rangle\rangle \\
& \longrightarrow\langle\langle 1>0 \Rightarrow 1:\{x \text { :nat } \mid x>0\}\rangle\rangle \\
& \longrightarrow *\langle\langle\text { true } \Rightarrow 1:\{x \text { :nat } \mid x>0\}\rangle\rangle \\
& \longrightarrow 1
\end{aligned}
$$

Each reduction step is explained as follows:

(1) Refinement predicates in the source type are peeled off until the type becomes a nonrefinement type. Those predicates are irrelevant to the check.

(2) If the source type is not a refinement type and the target type is a refinement type, then a cast turns into a waiting check, which is an intermediate state before actual checking of the predicate-in this case, $x>0$-in the target type. This intermediate state is required because, in general, the target type of a cast can be a nested refinement type like (1: int $\triangleright\{x:\{y$ :nat $\mid 1<y\} \mid x<5\})$. In this case, the subject of a waiting check becomes ( 1 : int $\triangleright\{y$ :nat $\mid 1<y\})$, which checks the predicate in the inner refinement type.

(3) A reflexive cast from nat to nat is eliminated. It is technically important that a reflexive cast disappears only when the source and target types are ground.

(4) After the subject of a waiting check becomes a value, an active check runs the predicate (obtained by substituting the value for the variable).

(5) The subject of an active check is evaluated.

(6) If the subject of an active check becomes true, the cast succeeds and returns the cast value.

A cast to fail takes similar steps; only the last step is different.

$$
\begin{aligned}
& (1:\{x \text { :nat } \mid x=1\} \triangleright\{x \text { :nat } \mid x>1\}) \\
& \longrightarrow * \text { false } \Rightarrow 1:\{x \text { :nat } \mid x>1\}\rangle\rangle \longrightarrow \text { blame }
\end{aligned}
$$

One might notice the refinement type in an active check is not used. It is just a type annotation like one in an abstraction, which is not used at run time, either.

Example 3.9. As we have explained in Section 1, a cast between (dependent) function types just wraps the function being cast by two split casts for inputs and outputs as follows.

$$
\begin{array}{r}
(\text { fix } f(x: \text { nat }): \text { nat. } x:(x \text { :nat }) \rightarrow \text { nat } \triangleright(y: \text { nat }) \rightarrow \\
\text { fix } y(y: \text { nat }):\{z: \text { nat } \mid z>y\} .(f i x x(x \text { :nat }):\{z \text { :nat } \mid z>y\} . \\
((\text { fix } f(x: \text { nat }): \text { nat. } x) x: \text { nat } \triangleright\{z \text { nat } \mid z>y\})) \\
(y: \text { nat } \triangleright \text { nat })
\end{array}
$$




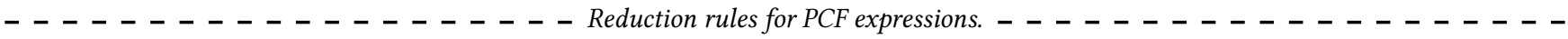

$$
\begin{aligned}
& (\mathrm{fix} f(x: \sigma): \tau . M) V \stackrel{\Delta}{\longrightarrow} M[x:=V][f:=\mathrm{fix} f(x: \sigma): \tau . M](\mathrm{R}-\mathrm{BETA}) \quad(\lambda \alpha . M) \Phi \stackrel{\Delta}{\longrightarrow} M[\alpha:=\Phi] \text { (R-SIGMA) } \\
& \operatorname{pred}(\operatorname{succ}(\bar{n})) \stackrel{\Delta}{\longrightarrow} \bar{n}(\mathrm{R}-\mathrm{PRED}) \quad \text { iszero }(0) \stackrel{\Delta}{\longrightarrow} \text { true }(\mathrm{R}-\text { IsZeroT) } \quad \text { iszero }(\operatorname{succ}(\bar{n})) \stackrel{\Delta}{\longrightarrow} \text { false (R-IsZERoF) } \\
& \text { if true then } M \text { else } N \stackrel{\Delta}{\longrightarrow} M(\mathrm{R} \text {-IFT) } \quad \text { if false then } M \text { else } N \stackrel{\Delta}{\longrightarrow} N \text { (R-IFF) }
\end{aligned}
$$

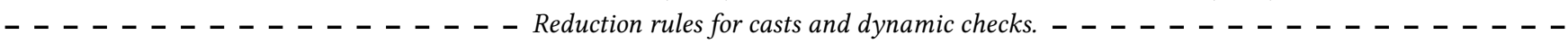

$$
\begin{aligned}
& (V: \text { bool } \triangleright \text { bool }) \stackrel{\Delta}{\longrightarrow} V \text { (R-CBool }) \quad(V: \text { nat } \triangleright \text { nat }) \stackrel{\Delta}{\longrightarrow} V \text { (R-CNAT) } \quad(V: \forall \alpha . \sigma \triangleright \forall \alpha . \tau) \stackrel{\Delta}{\longrightarrow} \lambda \alpha .(V \alpha: \sigma \triangleright \tau)(\mathrm{R}-\mathrm{CFORALL})
\end{aligned}
$$

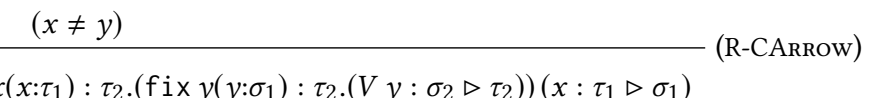

$$
\begin{aligned}
& (V:\{x: \sigma \mid M\} \triangleright \tau) \stackrel{\Delta}{\longrightarrow}(V: \sigma \triangleright \tau) \text { (R-CForget) } \quad \frac{\left(\sigma \neq\left\{x^{\prime}: \tau^{\prime} \mid M^{\prime}\right\}\right)}{(V: \sigma \triangleright\{x: \tau \mid M\}) \stackrel{\Delta}{\longrightarrow}\langle(V: \sigma \triangleright \tau) ?\{x: \tau \mid M\}\rangle)} \text { (R-PRECHECK) } \\
& \langle\langle V ?\{x: \tau \mid M\}\rangle \stackrel{\Delta}{\longrightarrow}\langle\langle M[x:=V] \Rightarrow V:\{x: \tau \mid M\}\rangle \text { (R-Снеск) } \quad\langle\langle\text { true } \Rightarrow V:\{x: \tau \mid M\}\rangle \stackrel{\Delta}{\longrightarrow} V \text { (R-Good) } \\
& \langle\langle\text { false } \Rightarrow V:\{x: \tau \mid M\}\rangle) \stackrel{\Delta}{\longrightarrow} \text { blame (R-BAD) } \\
& \text { - - - - - - - - - - - - - - - - - Reduction rules for coordinated choice. - - - - - - - - - - - - - - - - - } \\
& \left(M \|^{\Phi} N\right) \stackrel{\Delta \cup\left\{\Phi_{+}\right\}}{\longrightarrow} M(\mathrm{R}-\text { WorldL }) \quad\left(M \|^{\Phi} N\right) \stackrel{\Delta \cup\left\{\Phi_{-}\right\}}{\longrightarrow} N(\mathrm{R}-\text { WorldR }) \quad \mathcal{E}\left[\left(M_{1} \|^{\Phi} M_{2}\right)\right] \stackrel{\Delta}{\longrightarrow}\left(\mathcal{E}\left[M_{1}\right] \|^{\Phi} \mathcal{E}\left[M_{2}\right]\right)(\mathrm{R}-\mathrm{BRANCH})
\end{aligned}
$$

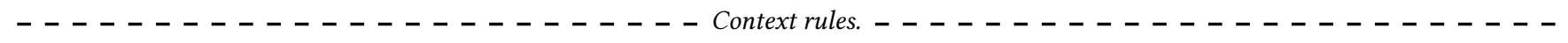

$$
\begin{aligned}
& \frac{M \stackrel{\Delta}{\longrightarrow} N}{\mathcal{E}[M] \stackrel{\Delta}{\longrightarrow} \mathcal{E}[N]}(\mathrm{R}-\mathrm{CTx}) \quad \mathcal{E}[\text { blame }] \stackrel{\Delta}{\longrightarrow} \text { blame (R-BlAmE) } \\
& \frac{M \stackrel{\Delta \cup\left\{\Phi_{+}\right\}}{\longrightarrow} M^{\prime}}{\left(M \|^{\Phi} N\right) \stackrel{\Delta}{\longrightarrow}\left(M^{\prime} \|^{\Phi} N\right)} \text { (R-ChoiceL) } \quad \frac{N \stackrel{\Delta \cup\left\{\Phi_{-}\right\}}{\longrightarrow} N^{\prime}}{\left(M \|^{\Phi} N\right) \stackrel{\Delta}{\longrightarrow}\left(M \|^{\Phi} N^{\prime}\right)} \text { (R-ChoiceR) }
\end{aligned}
$$

Figure 3: Operational semantics.

$$
\begin{array}{cr}
\Delta \mid=V \text { (Res-Value) } & \Delta \mid=b l a m e \text { (Res-Blame) } \\
\Delta \uplus\left\{\Phi_{+}\right\}\left|=R_{1} \quad \Delta \uplus\left\{\Phi_{-}\right\}\right|=R_{2} & \text { (Res-Choice) } \\
\Delta=\left(R_{1} \|^{\Phi} R_{2}\right)
\end{array}
$$

\section{Figure 4: Results in an orthant.}

Thus, blame is not immediately yielded, though the original cast is semantically incorrect. An actual check happens when the wrapped function is used as follows.

$$
\begin{aligned}
& \text { (fix } y(y \text { :nat) }:\{z \text { :nat } \mid z>y\} \text {.(fix } x(x \text { :nat }):\{z \text { :nat } \mid z>y\} \text {. } \\
& ((\text { fix } f(x \text { :nat }): \text { nat. } x) x \text { : nat } \triangleright\{z \text { :nat } \mid z>y\})) \\
& (y: \text { nat } \triangleright \text { nat })) 0 \\
& \longrightarrow^{*}(0: \text { nat } \triangleright\{z: \text { nat } \mid z>0\}) \\
& \longrightarrow \text { blame }
\end{aligned}
$$

Lastly, the rules for coordinated choice is as discussed in Section 2.

Definition 3.10 (Multi-step reduction). Given a fixed orthant $\Delta$, the multi-step reduction relation $M \stackrel{\Delta}{\longrightarrow} * N$ is defined as the reflexive and transitive closure of $\stackrel{\Delta}{\longrightarrow}$.

We define the notion of results below. Actually, a raw result may not be a normal form: For example, $\left(\left(\|^{\Phi} 2\right) \|^{\Phi} 3\right) \stackrel{\{\}}{\longrightarrow}\left(1 \|^{\Phi} 3\right)$. In other words, it depends on an orthant whether a given raw result is a normal form.
Definition 3.11 (Results in an orthant). A raw result $R$ is an actual result in an orthant $\Delta$ iff $\Delta=R$ can be derived by the rules in Figure 4.

\subsection{Type system}

The type system of $\lambda^{\mathrm{H} \|^{\Phi}}$ mainly consists of two judgments: the type well-formedness relation $\Gamma \vdash^{\Delta} \tau$, read "type $\tau$ is well-formed under environment $\Gamma$ in orthant $\Delta^{\prime}$; and the typing relation $\Gamma \vdash^{\Delta} M: \tau$, read "expression $M$ is given type $\tau$ under environment $\Gamma$ in orthant $\Delta$." Both judgments are annotated with an orthant $\Delta$, which is used in the type equivalence and reduction relations in premises of the typing rules. The derivation rules, which are mostly adapted from Sekiyama et al. [2015], are given in Figure 5; where nonzero is an abbreviation for $\{x$ :nat $\mid$ if iszero $(x)$ then false else true $\}$. An environment $\Gamma$ occurring in a derivation is called well-formed environment.

3.3.1 Type well-formedness rules. The principal role of the type well-formedness rules is to check that predicate expressions in refinement types are well typed Boolean expressions. The main rule is (W-REFINE), which does this check. Other rules are for checking well-formedness of subterms. Note that (W-ARRow) has no condition on the domain type; usual conditions $x \notin \operatorname{dom}(\Gamma)$ and $\Gamma \vdash^{\Delta} \sigma$ are ensured by weakening rules (W-WEAKEN) and (W-CWEAKEN) (also see Lemma 3.13 below). 


$$
\begin{aligned}
& \text { - - - - - - - - - - - - - - - Well-formedness rules. - - - - - - - - - - - - - - - - - } \\
& \vdash^{\Delta} \text { bool (W-Bool) } \quad \vdash^{\Delta} \text { nat (W-NAT) } \frac{\Gamma, x: \sigma \vdash^{\Delta} \tau}{\Gamma \vdash^{\Delta}(x: \sigma) \rightarrow \tau} \text { (W-Arrow) } \frac{\Gamma, x: \tau \vdash^{\Delta} M: \text { bool }}{\Gamma \vdash^{\Delta}\{x: \tau \mid M\}} \text { (W-REFINE) } \\
& \frac{\Gamma, \alpha \vdash^{\Delta} \tau \quad \alpha \notin \mathrm{fsv}(\Delta)}{\Gamma \vdash^{\Delta} \forall \alpha . \tau}(\text { W-ForaLL) } \\
& \vdash^{\Delta} \text { true : bool (T-True) } \quad \vdash^{\Delta} \text { false : bool (T-False) } \quad \vdash^{\Delta} 0: \text { nat (T-Zero) } \frac{\Gamma \vdash^{\Delta} \tau \quad(x \notin \operatorname{dom}(\Gamma))}{\Gamma, x: \tau \vdash^{\Delta} x: \tau}(\mathrm{T} \text {-VAR) } \\
& \frac{\Gamma \vdash^{\Delta} M:(x: \sigma) \rightarrow \tau \quad \Gamma \vdash^{\Delta} N: \sigma}{\Gamma \vdash^{\Delta} M N: \tau[x:=N]}(\text { T-App }) \quad \frac{\Gamma \vdash^{\Delta} M: \forall \alpha \cdot \tau \quad(\mathrm{fsv}(\Phi) \subseteq \operatorname{sdom}(\Gamma))}{\Gamma \vdash^{\Delta} M \Phi: \tau[\alpha:=\Phi]} \text { (T-SApP) } \\
& \frac{\Gamma, f:(x: \sigma) \rightarrow \tau, x: \sigma \vdash^{\Delta} M: \tau}{\Gamma \vdash^{\Delta} \text { fix } f(x: \sigma): \tau . M:(x: \sigma) \rightarrow \tau} \text { (T-Fun) } \frac{\Gamma \vdash^{\Delta} M: \text { nat }}{\Gamma r^{\Delta} \operatorname{succ}(M): \text { nat }} \text { (T-Succ) } \quad \frac{\Gamma r^{\Delta} M: \overline{\text { nonzero }}}{\Gamma r^{\Delta} \operatorname{pred}(M): \text { nat }} \text { (T-Pred) } \\
& \frac{\Gamma \vdash^{\Delta} M: \text { nat }}{\Gamma \vdash^{\Delta} \text { iszero }(M): \text { bool }} \text { (T-IsZero) } \frac{\Gamma \vdash^{\Delta} L: \text { bool } \Gamma r^{\Delta} M: \tau \quad \Gamma r^{\Delta} N: \tau}{\Gamma \vdash^{\Delta} \text { if } L \text { then } M \text { else } N: \tau} \text { (T-If) } \\
& \frac{\Gamma, \alpha \vdash^{\Delta} M: \tau \quad \alpha \notin \operatorname{fsv}(\Delta)}{\Gamma \vdash^{\Delta} \lambda \alpha . M: \forall \alpha . \tau}(\mathrm{T}-\mathrm{SFun}) \quad \frac{\Gamma \vdash^{\Delta \cup\left\{\Phi_{+}\right\}} M: \tau \quad \Gamma \vdash^{\Delta \cup\{\Phi+\}} N: \tau \quad \Gamma \vdash^{\Delta} \tau \quad(\mathrm{fsv}(\Phi) \subseteq \operatorname{sdom}(\Gamma))}{\Gamma \vdash^{\Delta}\left(M \|^{\Phi} N\right): \tau}(\mathrm{T}-\mathrm{CHOICE}) \\
& \frac{\Gamma \vdash^{\Delta} M: \sigma \quad \Gamma \vdash^{\Delta} \tau \quad(\sigma \sim \tau)}{\Gamma \vdash^{\Delta}(M: \sigma \triangleright \tau): \tau}(\text { T-CAST })
\end{aligned}
$$

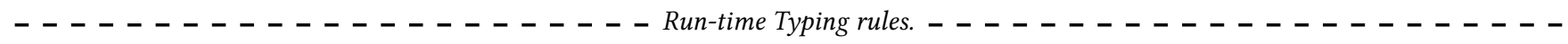

$$
\begin{aligned}
& \frac{\vdash^{\Delta} M: \tau \quad \vdash^{\Delta}\{x: \tau \mid N\}}{\vdash^{\Delta}\langle\langle M ?\{x: \tau \mid N\}\rangle\rangle:\{x: \tau \mid N\}}\left(\text { T-PreCheск) } \quad \frac{\vdash^{\Delta} M: \text { bool } \quad \vdash^{\Delta} V: \tau \quad \vdash^{\Delta}\{x: \tau \mid N\} \quad N[x:=V] \stackrel{\Delta}{\longrightarrow} *^{*} M}{\vdash^{\Delta}\langle\langle M \Rightarrow V:\{x: \tau \mid N\}\rangle:\{x: \tau \mid N\}}(\mathrm{T}-\mathrm{CHECK})\right. \\
& \frac{\vdash^{\Delta} \tau}{\vdash^{\Delta} \text { blame }: \tau} \text { (T-Blame) } \frac{\vdash^{\Delta} M: \sigma \quad \vdash^{\Delta} \tau \quad \sigma \triangleq \tau}{\vdash^{\Delta} M: \tau} \text { (T-Conv) } \frac{\vdash^{\Delta} V:\{x: \tau \mid M\}}{\vdash^{\Delta} V: \tau} \text { (T-ForgEt) } \\
& \frac{\vdash^{\Delta} V: \tau \quad \vdash^{\Delta}\{x: \tau \mid M\} \quad M[x:=V] \stackrel{\Delta}{\longrightarrow} * \text { true }}{\vdash^{\Delta} V:\{x: \tau \mid M\}} \text { (T-ExACT) } \\
& \text { - - - - - - - - - - - - - - - - - - Weakening rules. }
\end{aligned}
$$

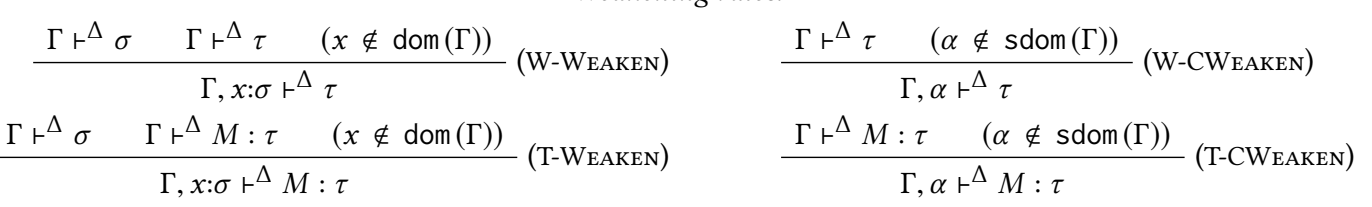

Figure 5: Type system.

3.3.2 Typing rules. The rule (T-PRED) demands that the operand of pred have the type $\{x$ :nat | if iszero $(x)$ then false else true $\}$, which enforces the argument to be nonzero so that pred $(M)$ can always be evaluated. The rule (T-APP) is a usual rule for applications in a dependent type system. The substitution in the conclusion of the rule expresses the dependency of the return type on actual arguments. The rule (T-SAPP) is for string applications, where fsv $(\Phi) \subseteq \operatorname{sdom}(\Gamma)$ checks that all string variables that appear are declared in the environment. The rule (T-CHOICE) is the heart of our type system and reflects the following intuition: a choice is well typed if its operands have the same type in corresponding orthants. The premise $\Gamma \vdash^{\Delta} \tau$ is required because the first two ensures only that $\tau$ is well-formed in orthants $\Delta \cup\left\{\Phi_{+}\right\}$and $\Delta \cup\left\{\Phi_{-}\right\}$ (see Lemma 4.9) but not necessarily in $\Delta$. The rule (T-CAST) is a standard one in manifest contract calculi, requiring the source and target types to be the same except for predicates. The requirement

$$
\begin{aligned}
& \text { bool } \sim \text { bool (C-BooL) } \quad \text { nat } \sim \text { nat }(\text { C-NAT) } \\
& \frac{\sigma_{1} \sim \tau_{1} \quad \sigma_{2} \sim \tau_{2}}{\left(x: \sigma_{1}\right) \rightarrow \sigma_{2} \sim\left(x: \tau_{1}\right) \rightarrow \tau_{2}} \text { (C-ARRow) } \\
& \frac{\sigma \sim \tau}{\{x: \sigma \mid M\} \sim \tau}\left(\text { C-ReFineL) } \quad \frac{\sigma \sim \tau}{\sigma \sim\{x: \tau \mid M\}}\right. \text { (C-ReFINER) } \\
& \frac{\sigma \sim \tau}{\forall \alpha . \sigma \sim \forall \alpha . \tau}(\text { C-FORALL) }
\end{aligned}
$$

Figure 6: Type compatibility.

is formalized as the type compatibility relation $\sigma \sim \tau$, defined by the rules in Figure 6. Since casts are only for dynamic contract checking, other kinds of differences than predicates are not allowed and so, $(M$ : bool $\triangleright$ int $)$ is rejected statically, for example.

The rules (T-PreCheck), (T-Check), (T-Blame), (T-Conv), (TForgET), and (T-ЕXАст) are distinguished as run-time typing rules, 
that is, those are not supposed to be used to typecheck source programs but required for type preservation (Lemma 4.10). This distinction makes a compile-time type checking decidable while some programs, like (fix $f(x:\{x$ :nat $\mid 1+1=2\}):\{x$ :nat $\mid 1+1=$ $2\} . x)(0$ : nat $\triangleright\{x$ :nat $\mid 2=2\})$, are rejected statically. Such a program is acceptable in the original work by Flanagan [2006], which uses subtyping, but Belo et al. [2011] start this style so that type checking of source programs does not rely on operational semantics.

Remark 3.12. Axioms, e.g, (W-Bool) and (T-True), demand that environments be empty. Thus, we have to use weakening rules to drop declarations before using these rules. As a consequence, all string variables and typed variables declared in a well-formed environment become distinct and types of typed variables become well-formed, as is stated in the following lemma.

Lemma 3.13 (WeLl-Formed ENVIRONMENTS). Suppose $\Gamma r^{\Delta} \tau$ or $\Gamma \vdash^{\Delta} M: \tau$.

- If $\Gamma=\Gamma^{\prime}, x: \sigma, \Gamma^{\prime \prime}$, then $x \notin \operatorname{dom}\left(\Gamma^{\prime}\right)$ and $\Gamma^{\prime} \vdash^{\Delta} \sigma$.

- If $\Gamma=\Gamma^{\prime}, \alpha, \Gamma^{\prime \prime}$, then $\alpha \notin \operatorname{sdom}\left(\Gamma^{\prime}\right)$.

By the lemma above, the rules (W-Arrow), (W-Refine), and (T-FUN) are free from checking well-formedness of the domain, underlying type, and argument type, respectively, and also conflict of bound variable names.

In contrast, conditions to avoid capture of string variables in an orthant are checked at the rules (W-ForALL) and (T-SFun). We also remark that the rules (W-CWEAKEN) and (T-CWEAKEN) work as if an additional declaration of a free string variable captures one in an orthant. See the derivation in Figure 7.

3.3.3 Type equivalence. A dependent type system usually uses a type equivalence relation to allow the type of an expression to be implicitly converted to an equal one implicitly. In many systems, type equivalence is based on full reduction of expressions but one in $\lambda^{\mathrm{H}} \|^{\Phi}$ is weaker. It is fine because all type conversions in a source program are due to explicit casts and its use is only to prove type preservation and value inversion. The type equivalence between $\sigma$ and $\tau$ in $\Delta$, written $\sigma \triangleq \partial$, is defined by rules in Figure 8, which follows Sekiyama et al. [2017]. Intuitively, two types are equivalent iff they are related by a sequence of common subexpression reduction, namely conversion from $M[x:=N]$ to $M\left[x:=N^{\prime}\right]$ in which $N \stackrel{\Delta}{\longrightarrow}$ $N^{\prime}$ holds. In fact, we have the following lemma.

LEMMA 3.14 (COMMON SUB-EXPRESSION REDUCTION MAKES EQUIVALENT TYPES). If $N \stackrel{\Delta}{\longrightarrow} N^{\prime}$, then $\tau[x:=N] \triangleq\left[\left[x:=N^{\prime}\right]\right.$.

As we have touched in Section 1, the type equivalence plays a crucial role to prove the type preservation property of $\lambda^{\mathrm{H} \|^{\Phi}}$. If $f$ has type $(x: \sigma) \rightarrow \tau$ and $M$ reduces to $M^{\prime}$, then $f M$ and $f M^{\prime}$ are given types $\tau[x:=M]$ and $\tau\left[x:=M^{\prime}\right]$, respectively. The type equivalence exactly captures this difference and (T-Conv) gives $f M^{\prime}$ type $\tau[x:=M]$, which is the type before reduction.

In $\lambda^{\mathrm{H} \|^{\Phi}}, f\left(M_{1} \|^{\Phi} M_{2}\right)$ reduces to $\left(f M_{1} \|^{\Phi} f M_{2}\right)$. In this case, showing type preservation is more tricky. The crux here is that type $\tau\left[x:=\left(M_{1} \|{ }^{\Phi} M_{2}\right)\right]$ is equivalent to $\tau\left[x:=M_{1}\right]$ in an orthant including $\Phi_{+}$and also to $\tau\left[x:=M_{2}\right]$ in an orthant including $\Phi_{-}$(but not equivalent to either of them in an orthant without them). So, $f M_{1}$ and $f M_{2}$ are given type $\tau\left[x:=\left(M_{1} \|^{\Phi} M_{2}\right)\right]$ in an orthant including $\Phi_{+}$and $\Phi_{-}$, respectively, and by the rule (T-Сногсе), $\left(f M_{1} \|^{\Phi} f M_{2}\right)$ is given type $\tau\left[x:=\left(M_{1} \|^{\Phi} M_{2}\right)\right]$. The following example shows a concrete example.

Example 3.15. Let $\Gamma=f:(x:$ bool $) \rightarrow\{z$ :bool $\mid x\}$. Then, $\left.\Gamma \vdash^{\{}\right\}$ $f$ (true $\|^{\text {foo }}$ false) $:\left\{z\right.$ :bool $\mid$ (true $\|^{\text {foo }}$ false) $\}$ is derived by (T-App). Since $f$ (true $\|^{\text {foo }}$ false) $\stackrel{\{\}}{\longrightarrow}\left(f\right.$ true $\|^{\text {foo }} f$ false), $\Gamma \vdash^{\{\}}\left(f\right.$ true $\|^{\text {foo }} f$ false $):\left\{z\right.$ :bool | (true $\|^{\text {foo }}$ false $\left.)\right\}$ must be derived for subject reduction. Actually, it can be derived as Figure 9. The point is $f$ true inhabits the orthant $\left\{f o_{+}\right\}$and

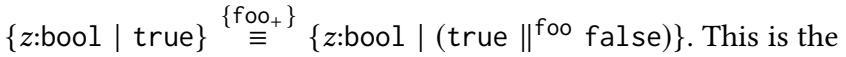
way our type system preserves an expression type before and after distributing a function argument.

\section{PROPERTIES OF $\lambda^{\mathrm{H} \|^{\Phi}}$}

Soundness properties of $\lambda^{\mathrm{H}} \|^{\Phi}$ consist of the familiar properties of type preservation (Lemma 4.10) and progress (Lemma 4.15) and also contract satisfaction (Lemma 4.14), which is briefly discussed in Section 1.

The last property deserves some more informal explanation. Suppose $M$ has type $\{x: \tau \mid N\}$ and all of its nondeterministic executions terminate. The result of evaluating $M$ is $R$, which is essentially a multi set of values and blame. Roughly speaking, the contract satisfaction says $R$ satisfies $N$ : for any value, denoted $V_{i}$, in $R$ satisfies the predicate $N$, that is, $N\left[x:=V_{i}\right]$ nondeterministically evaluates to true. Note that blame in $R$ is ignored because it denotes failed execution. The contract satisfaction property is a generalization of the value inversion property studied by Sekiyama et al. [2015] to the multi-valued setting of $\lambda^{\mathrm{H} \|^{\Phi}}$.

Remark 4.1. The phrase " $N\left[x:=V_{i}\right]$ nondeterministically evaluates to true" means that $N\left[x:=V_{i}\right] \stackrel{\Delta}{\longrightarrow}$ * true, where $\Delta$ is the orthant that $V_{i}$ inhabits. One might wonder if it is too strong to require a predicate $N$ to reduce to true, not to an expression like (true $\|^{\Phi} M$ ), even when $N$ can be nondeterministic. For example, consider type $\tau=\left\{x\right.$ :nat $\left.\mid\left(x=1 \|^{\Phi} x=2\right)\right\}$, whose predicate is nondeterministic, and a constant 1 ; then, $\left(x=1 \|^{\Phi} x=\right.$ 2) $[x:=1] \longrightarrow^{*}\left(\right.$ true $\|^{\Phi}$ false $) \neq$ true, which may look like a counterexample to contract satisfaction. Actually, it is not. First of all, $\vdash^{\{\}} 1: \tau$ cannot be derived because the type of 1 is only nat. We can make the expression well typed by inserting a cast. The judgment $\vdash^{\{\}}(1:$ nat $\triangleright \tau): \tau$ is derivable and this cast expression is reduced to $\left(1 \|^{\Phi}\right.$ blame). Now, the trick is to use the orthant that each value inhabit to run the predicate. So, the predicate is run in $\left\{\Phi_{+}\right\}$for 1 and we get $\left(x=1 \|^{\Phi} x=2\right)[x:=1] \stackrel{\left\{\Phi_{+}\right\}}{\longrightarrow}$ true.

We start with the following lemma, which is used everywhere in proofs and states a judgment that holds in some orthant holds in a "sub-orthant." For this lemma, we allow nonsensical orthants (see Remark 2.1).

Lemma 4.2 (Orthant weakening). Suppose $\Delta \subseteq \Delta^{\prime}$.

(1) If $M \stackrel{\Delta}{\longrightarrow} N$, then $M \stackrel{\Delta^{\prime}}{\longrightarrow} N$.

(2) If $M \triangleq N$, then $M \stackrel{\Delta^{\prime}}{\triangleq} N$. 


$$
\begin{aligned}
& \frac{r^{\left\{\alpha_{+}\right\}} \text {true : bool }}{\alpha r^{\left\{\alpha_{+}\right\}} \text {true : bool }} \text { (T-CWEAKEN) } \frac{r^{\left\{\alpha_{-}\right\}} \text {false : bool }}{\alpha r^{\left\{\alpha_{-}\right\}} \text {false : bool }} \text { (T-CWEAKen) } \frac{r^{\{\}} \text {bool }}{\alpha r^{\{\}} \text {bool }} \text { (W-CWEAKEN) } \\
& \frac{\alpha r^{\{\}}\left(\text {true } \|^{\alpha} \text { false }\right): \text { bool }}{r^{\{\}} \lambda \alpha . \text { (true } \|^{\alpha} \text { false) }: \forall \alpha \text {.bool }}(\text { T-SFun })
\end{aligned}
$$

Figure 7: Example of typing capturing a free variable in an orthant in the middle.

$$
\begin{aligned}
& \text { bool } \triangleq \text { bool (CSR-BooL }) \quad \text { nat } \triangleq \text { nat }(\text { CSR-NAT }) \\
& \left.\frac{\tau_{1} \triangleq \sigma_{1} \quad \tau_{2} \triangleq \sigma_{2}}{\left(x: \tau_{1}\right) \rightarrow \tau_{2} \triangleq\left(x: \sigma_{1}\right) \rightarrow \tau_{2}} \text { (CSR-ARROW }\right) \\
& \frac{\tau \stackrel{\triangleq}{\triangleq} \sigma}{\forall \alpha . \tau \triangleq \forall} \triangleq(\text { CSR-ForaLL }) \\
& \frac{\tau \stackrel{\Delta}{\triangleq} \sigma \stackrel{\Delta}{\longrightarrow} N^{\prime}}{\{x: \tau \mid M[y:=N]\} \triangleq\left\{x: \sigma \mid M\left[y:=N^{\prime}\right]\right\}} \text { (CSR-REFINEL) } \\
& \frac{\tau \stackrel{\Delta}{\triangleq} \sigma \quad N \stackrel{\Delta}{\longrightarrow} N^{\prime}}{\left\{x: \tau \mid M\left[y:=N^{\prime}\right]\right\} \triangleq\{x: \sigma \mid M[y:=N]\}} \text { (CSR-RefineR) }
\end{aligned}
$$

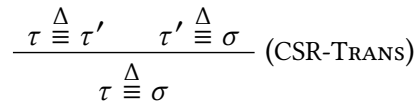

Figure 8: Type equivalence.

(3) If $\Gamma \vdash^{\Delta} \tau$, then $\Gamma \vdash^{\Delta^{\prime}} \tau$.

(4) If $\Gamma \vdash^{\Delta} M: \tau$, then $\Gamma \vdash^{\Delta^{\prime}} M: \tau$.

\subsection{Co-termination and evaluation}

The following lemma is important for a manifest contract calculus. It guarantees that type equivalence preserves the meaning of predicates (c.f. (CSR-REFINEL) and (CSR-REFINER)).

Definition 4.3 (Normal orthant). An orthant $\Delta$ is normal if and only if $\left\{\Phi_{+}, \Phi_{-}\right\} \nsubseteq \Delta$ for any $\Phi$.

LEMMA 4.4 (CO-TERMINATION FOR TRUE). Suppose $\Delta$ is normal and $N \stackrel{\Delta}{\longrightarrow} N^{\prime}$. Then $M[x:=N] \stackrel{\Delta}{\longrightarrow}$ * true $\Longleftrightarrow M[x:=$ $\left.N^{\prime}\right] \stackrel{\Delta}{\longrightarrow} *$ true.

This is a technical reason for which we develop the orthantbased reduction of $\lambda^{\mathrm{H} \|^{\Phi}}$. If we adopted a naive choice semantics $\left(M \|^{\Phi} N\right) \longrightarrow M$ and $\left(M \|^{\Phi} N\right) \longrightarrow N$ (regardless of orthants), we would have (true $\|^{\Phi}$ false) $\longrightarrow$ false and $x\left[x:=\left(\right.\right.$ true $\|^{\Phi}$ false $) \longrightarrow$ true and $x[x:=$ false $] \longrightarrow$ false, breaking the property. Actually, the same problem still occurs in nonsensical orthants, so we consider only the normal orthants, excluding nonsensical orthants.

Lemma 4.4 is, however, not easy to prove directly since our operational semantics is nondeterministic even if $\Delta$ is restricted to be normal. Instead, we give an alternative semantics, which is big-step and deterministic, and prove the property via this semantics. Figure 10 defines the alternative semantics for $\lambda^{\mathrm{H}} \|^{\Phi}$ in a big-step style. Most of the rules are straightforward; the key rules are (E-CHOICE),
(E-WorLdL), and (E-WorLdR), which correspond to (R-CHOICEL), (R-ChoiceR), (R-WorldL), and (R-WorldR). Note that (E-CHoice) uses disjoint union: both branches of a choice are evaluated only if they are not bogus. The following lemmas guarantee determinism and the two semantics agree with each other.

LemmA 4.5. If $M \Downarrow^{\Delta} R_{1}$ and $M \Downarrow^{\Delta} R_{2}$, then $R_{1}=R_{2}$.

LEMMA 4.6. If $\Delta$ is normal, $M \stackrel{\Delta}{\longrightarrow} *$, and $\Delta=R$, then $M \Downarrow^{\Delta} R$.

Lemma 4.7. If $M \Downarrow^{\Delta} R$, then $M \stackrel{\Delta}{\longrightarrow} *$ R.

Now, Lemma 4.4 follows from the following lemma, which can be (thanks to the alternative semantics) easily proven by routine induction on the given derivation of $M[x:=N] \Downarrow^{\Delta} R$.

Lemma 4.8 (Co-Termination). If $\Delta$ is normal, $N \stackrel{\Delta}{\longrightarrow} N^{\prime} \vee$ $N^{\prime} \stackrel{\Delta}{\longrightarrow} N$, and $M[x:=N] \Downarrow^{\Delta} R$, then there exists $R^{\prime}$ such that $R=R^{\prime}[x:=N]$ and $M\left[x:=N^{\prime}\right] \Downarrow^{\Delta} R^{\prime}\left[x:=N^{\prime}\right]$.

\subsection{Soundness of the type system}

Now, we show soundness properties. First, a usual type preservation property holds of $\lambda^{\mathrm{H} \|^{\Phi}}$.

Lemma 4.9 (Typing Regularity). If $\Gamma \vdash^{\Delta} M: \tau$, then $\Gamma \vdash^{\Delta} \tau$.

LEMMA 4.10 (TYPE PRESERVATION). If $\vdash^{\Delta} M: \tau$ and $M \stackrel{\Delta}{\longrightarrow} N$, then $r^{\Delta} N: \tau$.

Next, we show a value inversion lemma and then the contract satisfaction lemma by lifting the former result pointwise.

Definition 4.11 (Refinements). The set of expressions refining $\tau$, written $\operatorname{refines}(\tau)$, is defined as follows.

$\operatorname{refines}($ bool $)=\operatorname{refines}($ nat $)=\operatorname{refines}((x: \sigma) \rightarrow \tau)=\{\}$

$$
\operatorname{refines}(\{x: \tau \mid M\})=\{(x) M\} \cup \operatorname{refines}(\tau)
$$

LeMma 4.12 (VAlue inVERsion). If $\vdash^{\Delta} V: \tau$ and $\Delta$ is normal, then $M[x:=V] \stackrel{\Delta}{\longrightarrow} *$ true for all $(x) M \in \operatorname{refines}(\tau)$.

Definition 4.13. Result $R$ satisfies refinement type $\{x: \tau \mid M\}$ in orthant $\Delta$, written $R=^{\Delta}\{x: \tau \mid M\}$, if the judgment is derived by the following rules.

$$
\begin{aligned}
& \frac{M[x:=V] \stackrel{\Delta}{\longrightarrow} * \text { true }}{V \models^{\Delta}\{x: \tau \mid M\}} \quad \text { blame } \models^{\Delta}\{x: \tau \mid M\} \\
& \frac{R_{1}\left|=^{\Delta \cup\left\{\Phi_{+}\right\}}\{x: \tau \mid M\} \quad R_{2}\right|^{\Delta \cup\left\{\Phi_{-}\right\}}\{x: \tau \mid M\}}{\left.\left(R_{1} \|^{\Phi} R_{2}\right)\right|^{\Delta}\{x: \tau \mid M\}}
\end{aligned}
$$

LEMMA 4.14 (CONTRACT SATISFACTION). If $\Delta$ is normal and $\mathrm{r}^{\Delta}$ $R:\{x: \tau \mid M\}$, then $\left.R\right|^{\Delta}\{x: \tau \mid M\}$. 


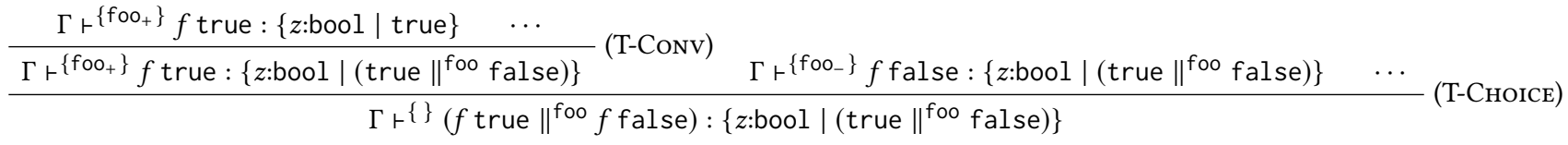

Figure 9: Example of typing for an expression after distributing an function argument.

$$
\begin{aligned}
& \text { true } \Downarrow^{\Delta} \text { true (E-True) false } \Downarrow^{\Delta} \text { false (E-False) } \quad 0 \Downarrow^{\Delta} 0 \text { (E-Zero) } \quad \text { fix } f(x: \sigma): \tau . M \Downarrow^{\Delta} \text { fix } f(x: \sigma): \tau . M \text { (E-Fun) } \\
& \lambda \alpha . M \Downarrow^{\Delta} \lambda \alpha . M \text { (E-SFun) } \frac{M \Downarrow^{\Delta} \bar{n}}{\operatorname{succ}(M) \Downarrow^{\Delta} \operatorname{succ}(\bar{n})}\left(\text { E-Succ) } \frac{M \Downarrow^{\Delta} \operatorname{succ}(\bar{n})}{\operatorname{pred}(M) \Downarrow^{\Delta} \bar{n}} \text { (E-Pred) } \frac{M \Downarrow^{\Delta} 0}{\text { iszero }(M) \Downarrow^{\Delta} \text { true }}\right. \text { (E-IsZERoT) } \\
& \frac{M \Downarrow^{\Delta} \operatorname{succ}(\bar{n})}{\text { iszero }(M) \Downarrow^{\Delta} \text { false }}(\text { E-IsZeroF }) \quad \frac{M \Downarrow^{\Delta} \text { fix } f(x: \sigma): \tau \cdot M_{1} \quad N \Downarrow^{\Delta} V_{1} \quad M_{1}\left[x:=V_{1}\right]\left[f:=\text { fix } f(x: \sigma): \tau \cdot M_{1}\right] \Downarrow^{\Delta} R}{M N \Downarrow^{\Delta} R} \text { (E-Beta) }
\end{aligned}
$$

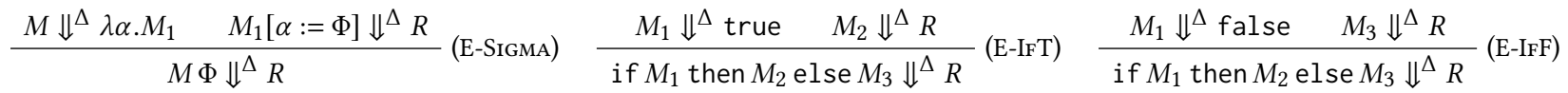

$$
\begin{aligned}
& \left.\frac{M \Downarrow^{\Delta} V}{(M: \text { bool } \triangleright \text { bool }) \Downarrow^{\Delta} V} \text { (E-CBool }\right) \quad \frac{M \Downarrow^{\Delta} V}{(M: \text { nat } \triangleright \text { nat }) \Downarrow^{\Delta} V} \text { (E-CNAT) } \frac{M \Downarrow^{\Delta} V}{(M: \forall \alpha . \sigma \triangleright \forall \alpha . \tau) \Downarrow^{\Delta} \lambda \alpha .(V \alpha: \sigma \triangleright \tau)} \text { (E-CForALL) } \\
& \frac{M \Downarrow^{\Delta} V \quad(x \neq y)}{\left(M:\left(y: \sigma_{1}\right) \rightarrow \sigma_{2} \triangleright\left(x: \tau_{1}\right) \rightarrow \tau_{2}\right) \Downarrow^{\Delta} \mathrm{fix} x\left(x: \tau_{1}\right): \tau_{2} \cdot\left(\mathrm{fix} y\left(y: \sigma_{1}\right): \tau_{2} \cdot\left(V y: \sigma_{2} \triangleright \tau_{2}\right)\right)\left(x: \tau_{1} \triangleright \sigma_{1}\right)} \text { (E-CARRow) } \\
& \frac{M \Downarrow^{\Delta} V \quad(V: \sigma \triangleright \tau) \Downarrow^{\Delta} R}{(M:\{x: \sigma \mid N\} \triangleright \tau) \Downarrow^{\Delta} R}(\text { E-CForget }) \quad \frac{M \Downarrow^{\Delta} V \quad\langle(V: \sigma \triangleright \tau) ?\{x: \tau \mid N\}\rangle \Downarrow^{\Delta} R \quad\left(\sigma \neq\left\{x^{\prime}: \tau^{\prime} \mid M^{\prime}\right\}\right)}{(M: \sigma \triangleright\{x: \tau \mid N\}) \Downarrow^{\Delta} R} \text { (E-CPRECHECK) } \\
& \frac{M \Downarrow^{\Delta} V \quad\left\langle\langle N[x:=V] \Rightarrow V:\{x: \tau \mid N\}\rangle \Downarrow^{\Delta} R\right.}{\langle\langle M ?\{x: \tau \mid N\}\rangle\rangle \Downarrow^{\Delta} R} \text { (E-Снеск) } \frac{M \Downarrow^{\Delta} \text { true }}{\left\langle\langle M \Rightarrow V:\{x: \tau \mid N\}\rangle \Downarrow^{\Delta} V\right.} \text { (E-Good) } \\
& \frac{M \Downarrow^{\Delta} \text { false }}{\left\langle\langle M \Rightarrow V:\{x: \tau \mid N\}\rangle \Downarrow^{\Delta}\right. \text { blame }}\left(\text { E-Bad) } \quad \text { blame } \Downarrow^{\Delta} \text { blame (E-Blame) } \frac{M \Downarrow^{\Delta} \text { blame } \quad(\mathcal{E} \neq V[])}{\mathcal{E}[M] \Downarrow^{\Delta} \text { blame }}\right. \text { (E-CтxBlame) } \\
& \frac{M \Downarrow^{\Delta}\left(R_{1} \|^{\Phi} R_{2}\right) \quad\left(\mathcal{E}\left[R_{1}\right] \|^{\Phi} \mathcal{E}\left[R_{2}\right]\right) \Downarrow^{\Delta} R \quad(\mathcal{E} \neq V[])}{\mathcal{E}[M] \Downarrow^{\Delta} R} \text { (E-Branch) } \frac{M \Downarrow^{\Delta} V \quad N \Downarrow^{\Delta} \text { blame }}{M N \Downarrow^{\Delta} \text { blame }} \text { (E-CTxBlameA) } \\
& \frac{M \Downarrow^{\Delta} V \quad N \Downarrow^{\Delta}\left(R_{1} \|^{\Phi} R_{2}\right) \quad\left(V R_{1} \|^{\Phi} V R_{2}\right) \Downarrow^{\Delta} R}{M N \Downarrow^{\Delta} R} \text { (E-BranchA) } \frac{M_{1} \Downarrow^{\Delta \uplus\left\{\Phi_{+}\right\}} R_{1} \quad M_{2} \Downarrow^{\Delta \uplus\left\{\Phi_{-}\right\}} R_{2}}{\left(M_{1} \|^{\Phi} M_{2}\right) \Downarrow^{\Delta}\left(R_{1} \|^{\Phi} R_{2}\right)} \text { (E-ChoICE) } \\
& \frac{M_{1} \Downarrow^{\Delta \cup\left\{\Phi_{+}\right\}} R \quad\left(\Phi_{-} \notin \Delta\right)}{\left(M_{1} \|^{\Phi} M_{2}\right) \Downarrow^{\Delta \cup\left\{\Phi_{+}\right\}} R}\left(\text { E-WorLDL) } \frac{M_{2} \Downarrow^{\Delta \cup\left\{\Phi_{-}\right\}} R \quad\left(\Phi_{+} \notin \Delta\right)}{\left(M_{1} \|^{\Phi} M_{2}\right) \Downarrow^{\Delta \cup\left\{\Phi_{-}\right\}} R}\right. \text { (E-WorLDR) }
\end{aligned}
$$

Figure 10: Deterministic evaluation.

Lastly, we show the usual progress property. The proof depends on Lemma 4.14 since our pred $(M)$ is a partial operation.

Lemma 4.15 (Progress). If $\Delta$ is normal and $r^{\Delta} M: \tau$, then $M$ is a result in $\Delta$; or there exists $N$ such that $M \stackrel{\Delta}{\longrightarrow} N$.

We close the section by combining the results above into the following theorem.

Theorem 4.16 (Soundness). If $\vdash^{\{\}} M: \tau$, then (1) $M \stackrel{\{\}}{\longrightarrow} * R$ and \{\} $\mid=R$; or (2) $M$ diverges. Moreover, if $\tau$ is a refinement type $\{x: \sigma \mid N\}$ and (1) holds, $R \mid=\{\}\{x: \sigma \mid N\}$.

\section{RELATED WORK}

Nondeterministic lambda calculi. Semantics of nondeterministic choice is studied from a long time ago [McCarthy 1961]. Well known semantics of nondeterministic choice is summarized by Søndergaard and Sestoft [1992]. Dezani-Ciancaglini et al. [1998] studied a calculus equipped with two of those semantics together, as parallelism and internal choice, and gave a fully abstract semantics to justify their operational semantics. Set-based reduction for choice was proposed by Kutzner and Schmidt-Schauß [1998] in order to guarantee confluence with nondeterministic choice. They also demonstrated that their language has the unfolding property [Søndergaard and Sestoft 1992]-a function application can be unfolded into a function body where actual arguments are substituted for formal (so the argument might be duplicated) without changing the meaning of the program; but what they actually proved is that an application to a "pure" expression can be unfolded, and they gave the method to discriminate pure and impure expressions. Therefore, their semantics does not suffice for our setting-we need semantics in which arbitrary expressions could duplicate.

There are few studies on dependent type systems for nondeterministic languages. In his manuscript, Warrell [2016] proposed a 
probabilistic dependent type system. His motivation is an investigation of Curry-Howard isomorphism for Markov logic network. As far as we understand, the type system has a (probabilistic) branching operator for types, as well as terms, and it appears that typechecking requires computation of normal forms. So, it is not clear how his method can be applied to Turing-complete languages.

Manifest contract calculi. Since the beginning of manifest contracts for hybrid type checking [Flanagan 2006], several extended calculi have been proposed: one with algebraic data types by Sekiyama et al. [2015]; one with stateful computation by Sekiyama and Igarashi [2017]; ones with parametric polymorphism [Belo et al. 2011; Sekiyama et al. 2017]; and SAGE [Gronski et al. 2006], a manifest contract calculus with recursive types, dynamic types, and the Type:Type discipline [Cardelli 1986]. We think that nondeterminism is an orthogonal issue and our approach is easy to integrate to those calculi.

One remark is that some studies [Knowles and Flanagan 2007; Sekiyama et al. 2015] use nondeterminism, but Sekiyama et al. [2015] force to make their operational semantics deterministic by assuming an oracle machine which chooses correct alternatives deterministically. Knowles and Flanagan [2007] use actual nondeterminism. However, they use nondeterministic expressions only for a type reconstruction, which includes predicate synthesis; and thus it is not intended that they appear in source code.

Static verification by using a dependent refinement type system. Although we give only dynamic checking in this paper, there are broad studies on static verification of higher-order functional programs [Cosman and Jhala 2017; Kobayashi et al. 2011; Rondon et al 2008; Terauchi 2010; Unno and Kobayashi 2009; Unno et al. 2018; Vazou et al. 2014; Zhu and Jagannathan 2013]. The main difference from a manifest contract calculus is that predicates of their refinement types are written in logical formulae. Thus, the decidability of their verification methods rely on that of the logic they adopt. It carries the trade-off: if logic is decidable, predicates are less expressive than the manifest way; or otherwise, predicates are indeed more expressive, but a verifier (compiler) makes false positives. A manifest contract system stays in the middle with paying run-time cost in exchange for no false positives. Note that hybrid type checking [Flanagan 2006]-the origin of manifest contract systems-combines static checking via subsumption rules; and some studies [Belo et al. 2011; Sekiyama et al. 2017] conjectured that up-casts, e.g., $(0:\{x$ :int $\mid x=0\} \triangleright\{x$ :int $\mid x \geq 0\})$, can be eliminated as optimization by defining a subtyping relation as an afterthought. So we would pay back some run-time cost even in a manifest contract system.

We should relate [Unno et al. 2018] in more detail. They proposed a verification method for a nondeterministic functional programming language. They avoid the difficulties discussed in this paper by restricting predicates in refinement types to be pure-actually, those are formulae of second-order arithmetic. The novelty in their type system is that it can expresses safety, non-safety, termination, and non-termination properties by using extended refinement types. A safety property means that the property holds in every possibility; and a non-safety property means that there exists a possibility in which the property holds. What we study in this paper can be viewed as a technique of dynamic verification of a non-safety property. It requires further work to see how static verification of casts (to see if each cast is successful) is related to Unno et al.'s static verification technique. Due to the nature of dynamic verification, termination verification is out of our scope.

Variational programming. Apart from nondeterminism, software product line (SPL) community develops a type system [Kästner et al. 2012] which deal with variation of code. They try to type check all variants of a single code-like $C$ code switching features by \#ifdef macro-without generating each variant code (because, in SPL engineering, the number of variants tends to be a quite large and it is hard to type check all variants separately). Their system has some similarity to our type system in the sense that a type of code varies according to the context it inhabits.

For a more theoretical aspect, Erwig and Walkingshaw [2011] study the choice calculus, a primitive model handling software variation. Since their work targets code variation, it has no operational semantics; but one extension [Chen et al. 2016] gave an operational semantics in order to manipulate variants at runtime. The semantics is quite similar in the point of synchronization of choice which is a one for code variants in their work (and not for nondeterminism); but other parts are fairly different and specialized for a specific purpose, e.g., their calculus has no mechanism to generate fresh choice points.

\section{CONCLUSION}

We have developed $\lambda^{\mathrm{H} \|^{\Phi}}$, a nondeterministic manifest contract calculus. By introducing a new kind of choice called coordinated choice, the calculus is free from restriction in the kind of programs used in predicates in refinements types.

Coordinated choice can share nondeterministic decisions by using names and the semantics is given in such a way that a program reduces to the set (expressed by $\|$ ) of all possible results. The semantics makes a choice copyable by substitution and solves problems that occur when we introduce dependent function types.

The semantics and type system of $\lambda^{\mathrm{H} \|^{\Phi}}$ are given by using the characteristic concept called orthant. Orthant gives the view for a choice. Since the view does not change the meaning of expressions except choices, we expect that coordinated choice is quite easily integrated into other manifest contract systems. By the observation, we hope this approach is easily applied to any other languages.

Future work. Coordinated choice we have proposed is demonic choice, that is, a choice diverges whenever one of its alternatives diverges. There is an opposite and more interesting choice, called angelic choice-a choice converges whenever one of its alternatives converges. McCarthy's amb operator [McCarthy 1961] is angelic choice. We would like to make coordinated choice angelic because not only is it theoretically interesting but also it is more useful in writing programs than demonic one. It is also interesting future work to investigate manifest contracts for the plural semantics, where different occurrences of the same variable can have different values.

\section{REFERENCES}

João Filipe Belo, Michael Greenberg, Atsushi Igarashi, and Benjamin C. Pierce. 2011. Polymorphic Contracts. In Proceedings of the 20th European Conference on Programming Languages and Systems: Part of the foint European Conferences on Theory and 
Practice of Software (ESOP'11/ETAPS'11). Springer-Verlag, Berlin, Heidelberg, 18-37. http://dl.acm.org/citation.cfm?id=1987211.1987213

Luca Cardelli. 1986. A Polymorphic $\lambda$-calculus with Type:Type. Technical Report 10. DEC Systems Research Center.

Sheng Chen, Martin Erwig, and Eric Walkingshaw. 2016. A Calculus for Variational Programming. In 30th European Conference on Object-Oriented Programming, ECOOP 2016, July 18-22, 2016, Rome, Italy. 6:1-6:28. https://doi.org/10.4230/LIPIcs.ECOOP. 2016.6

Benjamin Cosman and Ranjit Jhala. 2017. Local Refinement Typing. Proc. ACM Program Lang. 1, ICFP, Article 26 (Aug. 2017), 27 pages. https://doi.org/10.1145/3110270

Mariangiola Dezani-Ciancaglini, Ugo de'Liguoro, and Adolfo Piperno. 1998. A Filter Model for Concurrent lambda-Calculus. SIAM 7. Comput. 27, 5 (1998), 1376-1419. https://doi.org/10.1137/S0097539794275860

Martin Erwig and Eric Walkingshaw. 2011. The Choice Calculus: A Representation for Software Variation. ACM Trans. Softw. Eng. Methodol. 21, 1, Article 6 (Dec. 2011), 27 pages. https://doi.org/10.1145/2063239.2063245

Robert Bruce Findler and Matthias Felleisen. 2002. Contracts for Higher-order Functions. In Proceedings of the Seventh ACM SIGPLAN International Conference on Functional Programming (ICFP '02). ACM, New York, NY, USA, 48-59. https://doi.org/10.1145/581478.581484

Cormac Flanagan. 2006. Hybrid Type Checking. In Conference Record of the 33rd ACM SIGPLAN-SIGACT Symposium on Principles of Programming Languages (POPL '06) ACM, New York, NY, USA, 245-256. https://doi.org/10.1145/1111037.1111059

Michael Greenberg. 2015. Space-Efficient Manifest Contracts. In Proceedings of the 42Nd Annual ACM SIGPLAN-SIGACT Symposium on Principles of Programming Languages (POPL '15). ACM, New York, NY, USA, 181-194. https://doi.org/10.1145/ 2676726.2676967

Michael Greenberg, Benjamin C. Pierce, and Stephanie Weirich. 2010. Contracts Made Manifest. In Proceedings of the 37th Annual ACM SIGPLAN-SIGACT Symposium on Principles of Programming Languages (POPL '10). ACM, New York, NY, USA 353-364. https://doi.org/10.1145/1706299.1706341

Jessica Gronski, Kenneth Knowles, Aaron Tomb, Stephen N. Freund, and Cormac Flanagan. 2006. Sage: Hybrid checking for flexible specifications. In Scheme and Functional Programming Workshop. 93-104.

M. C. B. Hennessy and E. A. Ashcroft. 1977. Parameter-passing Mechanisms and Nondeterminism. In Proceedings of the Ninth Annual ACM Symposium on Theory of Computing (STOC '77). ACM, New York, NY, USA, 306-311. https://doi.org/10. $1145 / 800105.803420$

Christian Kästner, Sven Apel, Thomas Thüm, and Gunter Saake. 2012. Type Checking Annotation-based Product Lines. ACM Trans. Softw. Eng. Methodol. 21, 3, Article 14 (July 2012), 39 pages. https://doi.org/10.1145/2211616.2211617

Kenneth Knowles and Cormac Flanagan. 2007. Type Reconstruction for General Refinement Types. In Proceedings of the 16th European Symposium on Programming (ESOP'07). Springer-Verlag, Berlin, Heidelberg, 505-519. http://dl.acm.org/citation. cfm?id=1762174.1762220

Kenneth Knowles and Cormac Flanagan. 2010. Hybrid Type Checking. ACM Transactions on Programming Languages and Systems 32, 2:6 (2010). https://doi.org/10. 1145/1667048.1667051

Naoki Kobayashi, Ryosuke Sato, and Hiroshi Unno. 2011. Predicate abstraction and CEGAR for higher-order model checking. In Proceedings of the 32nd ACM SIGPLAN Conference on Programming Language Design and Implementation, PLDI 2011, San fose, CA, USA, fune 4-8, 2011. 222-233. https://doi.org/10.1145/1993498.1993525

Arne Kutzner and Manfred Schmidt-Schauß. 1998. A Non-deterministic Call-byneed Lambda Calculus. In Proceedings of the Third ACM SIGPLAN International Conference on Functional Programming (ICFP '98). ACM, New York, NY, USA, 324335. https://doi.org/10.1145/289423.289462

John McCarthy. 1961. A Basis for a Mathematical Theory of Computation, Preliminary Report. In Papers Presented at the May 9-11, 1961, Western foint IRE-AIEE-ACM Computer Conference (IRE-AIEE-ACM '61 (Western)). ACM, New York, NY, USA 225-238. https://doi.org/10.1145/1460690.1460715

Bertrand Meyer. 1988. Object-Oriented Software Construction, 1st edition. Prentice-Hall.

Robin Milner. 1999. Communicating and Mobile Systems: the $\pi$-Calculus. Cambridge University Press.

Andrew M. Pitts. 2000. Operational Semantics and Program Equivalence. In Proceedings of Applied Semantics, International Summer School, APPSEM 2000 (Lecture Notes on Computer Science), Vol. 2395. Springer-Verlag, 378-412.

Patrick M. Rondon, Ming Kawaguci, and Ranjit Jhala. 2008. Liquid Types. In Pro ceedings of the 29th ACM SIGPLAN Conference on Programming Language Design and Implementation (PLDI '08). ACM, New York, NY, USA, 159-169. https: //doi.org/10.1145/1375581.1375602

Taro Sekiyama and Atsushi Igarashi. 2017. Stateful manifest contracts. In Proceedings of the 44th ACM SIGPLAN Symposium on Principles of Programming Languages, POPL 2017, Paris, France, Fanuary 18-20, 2017. 530-544. http://dl.acm.org/citation. cfm?id=3009875

Taro Sekiyama, Atsushi Igarashi, and Michael Greenberg. 2017. Polymorphic Manifest Contracts, Revised and Resolved. ACM Trans. Program. Lang. Syst. 39, 1 (2017), 3:1-3:36. https://doi.org/10.1145/2994594
Taro Sekiyama, Yuki Nishida, and Atsushi Igarashi. 2015. Manifest Contracts for Datatypes. In Proceedings of the 42nd Annual ACM SIGPLAN-SIGACT Symposium on Principles of Programming Languages, POPL 2015, Mumbai, India, fanuary 15-17, 2015. 195-207. https://doi.org/10.1145/2676726.2676996

H. Søndergaard and P. Sestoft. 1992. Non-determinism in Functional Languages. Comput. F. 35, 5 (Oct. 1992), 514-523. https://doi.org/10.1093/comjnl/35.5.514

Tachio Terauchi. 2010. Dependent types from counterexamples. In Proceedings of the 37th ACM SIGPLAN-SIGACT Symposium on Principles of Programming Languages, POPL 2010, Madrid, Spain, January 17-23, 2010. 119-130. https://doi.org/10.1145/ 1706299.1706315

Hiroshi Unno and Naoki Kobayashi. 2009. Dependent Type Inference with Interpolants. In Proceedings of the 11th ACM SIGPLAN Conference on Principles and Practice of Declarative Programming (PPDP '09). ACM, New York, NY, USA, 277-288. https: //doi.org/10.1145/1599410.1599445

Hiroshi Unno, Yuki Satake, and Tachio Terauchi. 2018. Relatively complete refinement type system for verification of higher-order non-deterministic programs. PACMPL 2, POPL (2018), 12:1-12:29. https://doi.org/10.1145/3158100

Niki Vazou, Eric L. Seidel, Ranjit Jhala, Dimitrios Vytiniotis, and Simon Peyton-Jones. 2014. Refinement Types for Haskell. In Proceedings of the 19th ACM SIGPLAN International Conference on Functional Programming (ICFP '14). ACM, New York, NY, USA, 269-282. https://doi.org/10.1145/2628136.2628161

Philip Wadler and Robert Bruce Findler. 2009. Well-typed programs can't be blamed. In Proceedings of European Symposium on Programming (ESOP'09) (Lecture Notes on Computer Science). 1-16.

Jonathan H. Warrell. 2016. A Probabilistic Dependent Type System based on NonDeterministic Beta Reduction. CoRR abs/1602.06420 (2016). arXiv:1602.06420 http://arxiv.org/abs/1602.06420

Andrew K. Wright and Matthias Felleisen. 1994. A Syntactic Approach to Type Soundness. Information and Computation 115, 1 (Nov. 1994), 38-94.

He Zhu and Suresh Jagannathan. 2013. Compositional and Lightweight Dependent Type Inference for ML. In Verification, Model Checking, and Abstract Interpretation, 14th International Conference, VMCAI 2013, Rome, Italy, January 20-22, 2013. Proceedings. 295-314. https://doi.org/10.1007/978-3-642-35873-9_19 